\title{
Fermion mass and mixing pattern in a minimal T7 flavor 331 model.
}

\author{
A. E. Cárcamo Hernández圈 and R. Martinez畔 \\ ${ }^{a}$ Universidad Técnica Federico Santa María \\ and Centro Científico-Tecnológico de Valparaíso \\ Casilla 110-V, Valparaíso, Chile, \\ ${ }^{b}$ Universidad Nacional de Colombia, \\ Departamento de Física, Ciudad Universitaria, \\ Bogotá D.C., Colombia.
}

(Dated: September 22, 2018)

\begin{abstract}
We present a model based on the $S U(3)_{C} \otimes S U(3)_{L} \otimes U(1)_{X}$ gauge symmetry having an extra $T_{7} \otimes Z_{2} \otimes Z_{3} \otimes Z_{14}$ flavor group, which successfully describes the observed SM fermion mass and mixing pattern. In this framework, the light active neutrino masses arise via double seesaw mechanism and the observed charged fermion mass and quark mixing hierarchy is a consequence of the $Z_{2} \otimes Z_{3} \otimes Z_{14}$ symmetry breaking at very high energy. In our minimal $T_{7}$ flavor 331 model, the spectrum of neutrinos includes very light active neutrinos as well as heavy and very heavy sterile neutrinos. The model has in total 16 effective free parameters, which are fitted to reproduce the experimental values of the 18 physical observables in the quark and lepton sectors. The obtained physical observables for both quark and lepton sectors are compatible with their experimental values. The model predicts the effective Majorana neutrino mass parameter of neutrinoless double beta decay to be $m_{\beta \beta}=$ 3 and $40 \mathrm{meV}$ for the normal and the inverted neutrino spectrum, respectively. Furthermore, our model features a vanishing leptonic Dirac CP violating phase.
\end{abstract}

\section{INTRODUCTION}

In spite of the experimental confirmation of the big accomplishments of the Standard Model (SM) in describing electroweak phenomena, given by the discovery of the $\sim 126 \mathrm{GeV}$ Higgs boson by ATLAS and CMS collaborations at the CERN Large Hadron Collider (LHC) [1 [4], there are several issues that the SM is unable to address. Some of these issues are the Dark Matter problem, the fermion mass and mixing hierarchy and the neutrino oscillations [5, 6]. Furthermore, there are problems with the matter-antimatter asymmetry related with new phases responsible for CP violation. Moreover, the SM does not explain the tiny value of the neutron dipole moment. Because of these reasons it is necesary to consider an extension of the Standard Model. In particular, the observed the quark mass and mixing pattern, which cannot be explained from first principles in the context of the Standard Model, is a clear indication of physics beyond the Standard Model. On the other hand, the discovery of the Higgs boson opens the possibility to unravel the Electroweak Symmetry Breaking (EWSB) mechanism and motivates to study extensions of the SM having extra scalar particles that could provide an explanation for the existence of Dark Matter 7].

The lack of predictivity of the Standard Model Yukawa sector, motivates to consider extensions of the Standard Model aimed to address its flavor puzzle. Discrete flavor symmetries are important because they generate fermion textures useful to explain the three generation flavor structure, for recent reviews see Refs. 8 10$]$. These discrete flavour symmetries have been employed in extensions of the Standard Model with the aim to study the fermion mass and mixing hierarchy in order to address the flavor puzzle of the SM. Discrete flavor symmetries can arise from the underlying theory, e.g., string theory or compactification via orbifolding. In particular, from heterotic orbifold models, one can generate the $D_{4}$ and $\Delta(54)$ flavor symmetries [1]. Furthermore, magnetized/intersecting D-brane models can generate the $\Delta(27)$ flavor symmetry [1]]. Discrete symmetries may link the low energy physics and the underlying theory.

Furthermore, another unaswered issue in particle physics is the existence of three generations of fermions at low energies. The mixing patterns of leptons and quarks are significantly different; while in the quark sector, the mixing angles are small, in the lepton sector two of the mixing angles are large and one is small. Models having $S U(3)_{C} \otimes$ $S U(3)_{L} \otimes U(1)_{X}$ as a gauge symmetry, are vectorlike with three fermion generations and thus do not contain anomalies [12-16]. Defining the electric charge as the linear combination of the $T_{3}$ and $T_{8} S U(3)_{L}$ generators, we have that it

*Electronic address: antonio.carcamo@usm.cl

${ }^{\dagger}$ Electronic address: remartinezm@unal.edu.co 
is a free parameter, which does not depend on the anomalies $(\beta)$. The charge of the exotic particles is defined by setting a value for the $\beta$ parameter. Setting $\beta=-\frac{1}{\sqrt{3}}$, implies that the third component of the weak lepton triplet is a neutral field $\nu_{R}^{C}$ allowing to build the Dirac Yukawa term with the usual field $\nu_{L}$ of the weak doublet. By adding very heavy sterile neutrinos $N_{R}^{1,2,3}$ in the model, light neutrino masses can be generated via double seesaw mechanism. The 331 models with $\beta=-\frac{1}{\sqrt{3}}$ provide an alternative neutrino mass generation mechanism and include in their neutrino spectrum light active sub-eV scale neutrinos as well as sterile neutrinos which could be dark matter candidates if they are light enough or candidates for detection at the LHC, if they have $\mathrm{TeV}$ scale masses. Having TeV scale sterile neutrinos in its neutrino spectrum, makes the 331 models very important since if these sterile neutrinos are detected at the LHC, these models can shed light in the understanding of the electroweak symmetry breaking mechanism.

Neutrino oscillation experiments [6, 17 21] show that there are at most one massless active neutrino and that the different neutrino flavors mix. Neutrino oscillations experiments do not determine neither the absolute value of the neutrino masses nor the Majorana or Dirac feature of the neutrino. Nevertheless neutrino mass bounds can be obtained from tritio beta decay [22], double beta decay [23] and cosmology [24].

The global fits of the available data from the Daya Bay [17], T2K [18], MINOS [19], Double CHOOZ [20] and RENO 21] neutrino oscillation experiments, constrain the neutrino mass squared splittings and mixing parameters 25]. The current experimental data on neutrino mixing parameters suggests a violation of the tribimaximal symmetry described by the Tribimaximal Mixing (TBM) matrix, whose predicted mixing angles satisfy $\left(\sin ^{2} \theta_{12}\right)_{T B M}=\frac{1}{3}$,

$\left(\sin ^{2} \theta_{23}\right)_{T B M}=\frac{1}{2}$, and $\left(\sin ^{2} \theta_{13}\right)_{T B M}=0$. To generate nearly tribimaximal leptonic mixing angles consistent with the experimental data, discrete symmetry groups [26 35] are implemented in extensions of the Standard Model. Another approach to address the flavor puzzle consists in postulating fermion mass textures (see Ref [36] for some works considering textures). Moreover, models based on extended symmetries in the context of Multi-Higgs sectors, Grand Unification, Extradimensions and Superstrings have been explored [8, 37 40] to provide an explanation for the observed fermion mass and mixing pattern. Furthermore, in the framework of minimal 331 models, the discrete groups require of an extra high energy scale, larger than the scale of breaking of the $S U(3)_{C} \otimes S U(3)_{L} \otimes U(1)_{X}$ symmetry, and in some cases, new scalar fields need to be introduced at the very high discrete symmetry breaking scale, with the aim to fulfill the irreducible representations of these discrete groups that allow to get viable fermion textures at low energies, after the gauge and discrete symmetries are spontaneosly broken.

In this paper we formulate an extension of the minimal $S U(3)_{C} \times S U(3)_{L} \times U(1)_{X}$ model with $\beta=-\frac{1}{\sqrt{3}}$, where an extra $T_{7} \otimes Z_{2} \otimes Z_{3} \otimes Z_{14}$ discrete group (see Ref [35] for studies about the $T_{7}$ flavor group) extends the symmetry of the model and very heavy extra scalar fields are added with the aim to generate viable and predictive textures for the fermion sector that successfully describe the fermion mass and mixing pattern. The obtained physical observables in the quark and lepton sector are consistent with the experimental data. Our model at low energies reduces to the minimal 331 model with $\beta=-\frac{1}{\sqrt{3}}$.

The content of this paper goes as follows. In Sec. [II we describe the proposed model. Sec. [II] is devoted to the discussion of lepton masses and mixings. In Sec. IV] we present our results in terms of quark masses and mixing, which is followed by a numerical analysis. Conclusions are stated in Sec. V. Appendix A provides a brief description of the $T_{7}$ discrete group.

\section{THE MODEL}

\section{A. Particle content}

We consider a $S U(3)_{C} \otimes S U(3)_{L} \otimes U(1)_{X} \otimes T_{7} \otimes Z_{2} \otimes Z_{3} \otimes Z_{14}$ model where the full symmetry $\mathcal{G}$ is spontaneously broken in three steps as follows:

$$
\begin{aligned}
\mathcal{G}= & S U(3)_{C} \otimes S U(3)_{L} \otimes U(1)_{X} \otimes T_{7} \otimes Z_{2} \otimes Z_{3} \otimes Z_{14} \stackrel{\Lambda_{\text {int }}}{\longrightarrow} \\
& S U(3)_{C} \otimes S U(3)_{L} \otimes U(1)_{X} \stackrel{v_{\chi}}{\longrightarrow} S U(3)_{C} \otimes S U(2)_{L} \otimes U(1)_{Y} \stackrel{v_{\eta}, v_{\rho}}{\longrightarrow} \\
& S U(3)_{C} \otimes U(1)_{Q},
\end{aligned}
$$

where the hierarchy $v_{\eta}, v_{\rho} \ll v_{\chi} \ll \Lambda_{\text {int }}$ among the symmetry breaking scales is fullfilled.

The electric charge in our 331 model is defined as:

$$
Q=T_{3}-\frac{1}{\sqrt{3}} T_{8}+X I,
$$


where $T_{3}$ and $T_{8}$ are the $S U(3)_{L}$ diagonal generators and $I$ is the $3 \times 3$ identity matrix.

Two families of quarks are grouped in a $3^{*}$ irreducible representations (irreps), as required from the $S U(3)_{L}$ anomaly cancellation. Furthermore, from the quark colors, it follows that the number of $3^{*}$ irreducible representations is six. The other family of quarks is grouped in a 3 irreducible representation. Moreover, there are six 3 irreps taking into account the three families of leptons. Consequently, the $S U(3)_{L}$ representations are vector like and do not contain anomalies. The quantum numbers for the fermion families are assigned in such a way that the combination of the $U(1)_{X}$ representations with other gauge sectors is anomaly free. Therefore, the anomaly cancellation requirement implies that quarks are unified in the following $\left(S U(3)_{C}, S U(3)_{L}, U(1)_{X}\right)$ left- and right-handed representations:

$$
\begin{array}{cc}
Q_{L}^{1,2}=\left(\begin{array}{c}
D^{1,2} \\
-U^{1,2} \\
J^{1,2}
\end{array}\right)_{L}:\left(3,3^{*}, 0\right), & Q_{L}^{3}=\left(\begin{array}{c}
U^{3} \\
D^{3} \\
T
\end{array}\right)_{L}:(3,3,1 / 3), \\
D_{R}^{1,2,3}:(3,1,-1 / 3), & U_{R}^{1,2,3}:(3,1,2 / 3), \\
J_{R}^{1,2}:(3,1,-1 / 3), & T_{R}:(3,1,2 / 3) .
\end{array}
$$

Here $U_{L}^{i}$ and $D_{L}^{i}(i=1,2,3)$ are the left handed up- and down-type quarks in the flavor basis. The right handed SM quarks $U_{R}^{i}$ and $D_{R}^{i}(i=1,2,3)$ and right handed exotic quarks $T_{R}$ and $J_{R}^{1,2}$ are assigned into $S U(3)_{L}$ singlets representations, so that their $U(1)_{X}$ quantum numbers correspond to their electric charges.

Furthermore, cancellation of anomalies implies that leptons are grouped in the following $\left(S U(3)_{C}, S U(3)_{L}, U(1)_{X}\right)$ left- and right-handed representations:

$$
\begin{aligned}
& L_{L}^{1,2,3}=\left(\begin{array}{c}
\nu^{1,2,3} \\
e^{1,2,3} \\
\left(\nu^{1,2,3}\right)^{c}
\end{array}\right)_{L}:(1,3,-1 / 3), \\
& e_{R}:(1,1,-1), \quad \mu_{R}:(1,1,-1), \quad \tau_{R}:(1,1,-1), \\
& N_{R}^{1}:(1,1,0), \quad N_{R}^{2}:(1,1,0), \quad N_{R}^{3}:(1,1,0) .
\end{aligned}
$$

where $\nu_{L}^{i}$ and $e_{L}^{i}\left(e_{L}, \mu_{L}, \tau_{L}\right)$ are the neutral and charged lepton families, respectively. Let's note that we assign the right-handed leptons as $S U(3)_{L}$ singlets, which implies that their $U(1)_{X}$ quantum numbers correspond to their electric charges. The exotic leptons of the model are: three neutral Majorana leptons $\left(\nu^{1,2,3}\right)_{L}^{c}$ and three right-handed Majorana leptons $N_{R}^{1,2,3}$ (A recent discussion of double and inverse see-saw neutrino mass generation mechanisms in the context of 331 models can be found in Ref. [41]).

The scalar sector the 331 models includes: three 3's irreps of $S U(3)_{L}$, where one triplet $\chi$ gets a TeV scale vaccuum expectation value $(\mathrm{VEV}) v_{\chi}$, that breaks the $S U(3)_{L} \times U(1)_{X}$ symmetry down to $S U(2)_{L} \times U(1)_{Y}$, thus generating the masses of non SM fermions and non SM gauge bosons; and two light triplets $\eta$ and $\rho$ acquiring electroweak scale VEVs $v_{\eta}$ and $v_{\rho}$, respectively and thus providing masses for the fermions and gauge bosons of the SM.

Regarding the scalar sector of the minimal 331 model, we assign the scalar fields in the following $\left[S U(3)_{L}, U(1)_{X}\right]$ representations:

$$
\begin{aligned}
& \chi=\left(\begin{array}{c}
\chi_{1}^{0} \\
\chi_{2}^{-} \\
\frac{1}{\sqrt{2}}\left(v_{\chi}+\xi_{\chi} \pm i \zeta_{\chi}\right)
\end{array}\right):(3,-1 / 3), \quad \rho=\left(\begin{array}{c}
\rho_{1}^{+} \\
\left.\frac{1}{\sqrt{2}}\left(v_{\rho}+\xi_{\rho} \pm i \zeta_{\rho}\right)\right):(3,2 / 3), \\
\rho_{3}^{+}
\end{array}\right):(3,-1 / 3) . \\
& \eta=\left(\begin{array}{c}
\frac{1}{\sqrt{2}}\left(v_{\eta}+\xi_{\eta} \pm i \zeta_{\eta}\right) \\
\eta_{2}^{-} \\
\eta_{3}^{0}
\end{array}\right):
\end{aligned}
$$

We extend the scalar sector of the minimal 331 model by adding the following eleven very heavy $S U(3)_{L}$ scalar singlets:

$$
\sigma \sim(1,0), \quad \tau \sim(1,0), \quad \xi_{j}:(1,0), \quad \zeta_{j}:(1,0), \quad S_{j}:(1,0), \quad j=1,2,3 .
$$

We assign the scalars into $T_{7}$ triplet, $T_{7}$ antitriplet and $T_{7}$ singlet representions. The $T_{7} \otimes Z_{2} \otimes Z_{3} \otimes Z_{14}$ assignments of the scalar fields are:

$$
\begin{array}{llrl}
\eta \sim\left(\mathbf{1}_{0}, 1, e^{\frac{2 \pi i}{3}}, 1\right), & \rho \sim\left(\mathbf{1}_{0}, 1, e^{-\frac{2 \pi i}{3}}, 1\right), & \chi \sim\left(\mathbf{1}_{0}, 1,1,1\right), & \tau \sim\left(\mathbf{1}_{1},-1,1,1\right), \\
\xi \sim\left(3,1, e^{\frac{2 \pi i}{3}}, 1\right), & \zeta \sim(\overline{\mathbf{3}}, 1,1,1), & S \sim\left(\mathbf{3}, 1, e^{\frac{2 \pi i}{3}}, 1\right), & \sigma \sim\left(\mathbf{1}_{0}, 1,1, e^{-\frac{i \pi}{7}}\right) .
\end{array}
$$


It is noteworthy that the $S U(3)_{L}$ singlet scalar field $\tau$ is the only scalar odd under the $Z_{2}$ symmetry and assigned as a non trivial $T_{7}$ singlet. This scalar field $\tau$ is crucial for explaining the hierarchy between the SM up and SM down type quark masses.

In the concerning to the lepton sector, we have the following $T_{7} \otimes Z_{2} \otimes Z_{3} \otimes Z_{14}$ assignments:

$$
\begin{array}{ll}
L_{L} \sim\left(\mathbf{3}, 1, e^{-\frac{2 \pi i}{3}}, 1\right), & e_{R} \sim\left(\mathbf{1}_{0}, 1, e^{-\frac{2 \pi i}{3}},-1\right), \quad \mu_{R} \sim\left(\mathbf{1}_{1}, 1, e^{-\frac{2 \pi i}{3}}, e^{\frac{4 i \pi}{7}}\right), \\
\tau_{R} \sim\left(\mathbf{1}_{2}, 1, e^{-\frac{2 \pi i}{3}}, e^{\frac{2 i \pi}{7}}\right), & N_{R} \sim\left(\mathbf{3}, 1, e^{-\frac{2 \pi i}{3}}, 1\right),
\end{array}
$$

while the $T_{7} \otimes Z_{2} \otimes Z_{3} \otimes Z_{14}$ assignments for the quark sector are:

$$
\begin{array}{lll}
Q_{L}^{1} \sim\left(\mathbf{1}_{0}, 1,1, e^{\frac{2 \pi i}{7}}\right), & Q_{L}^{2} \sim\left(\mathbf{1}_{0}, 1,1, e^{\frac{\pi i}{7}}\right), & Q_{L}^{3} \sim\left(\mathbf{1}_{0}, 1,1,1\right), \\
U_{R}^{1} \sim\left(\mathbf{1}_{0}, 1, e^{-\frac{2 \pi i}{3}}, e^{\frac{2 \pi i}{7}}\right), & U_{R}^{2} \sim\left(\mathbf{1}_{0}, 1, e^{-\frac{2 \pi i}{3}}, e^{\frac{\pi i}{7}}\right), & U_{R}^{3} \sim\left(\mathbf{1}_{0}, 1, e^{-\frac{2 \pi i}{3}}, 1\right), \\
D_{R}^{1} \sim\left(\mathbf{1}_{0},-1, e^{\frac{2 \pi i}{3}}, e^{\frac{2 \pi i}{7}}\right), & D_{R}^{2} \sim\left(\mathbf{1}_{0},-1, e^{\frac{2 \pi i}{3}}, e^{\frac{\pi i}{7}}\right), & D_{R}^{3} \sim\left(\mathbf{1}_{0},-1, e^{\frac{2 \pi i}{3}}, 1\right), \\
T_{R} \sim\left(\mathbf{1}_{0}, 1,1,1\right), & J_{R}^{1} \sim\left(\mathbf{1}_{0}, 1,1, e^{\frac{2 \pi i}{7}}\right), \quad J_{R}^{2} \sim\left(\mathbf{1}_{0}, 1,1, e^{\frac{\pi i}{7}}\right) .
\end{array}
$$

Here the dimensions of the $T_{7}$ irreducible representations are specified by the numbers in boldface. In the concerning to the lepton sector, we recall that the left and right handed leptons are grouped into $T_{7}$ triplet and $T_{7}$ singlet irreducible representations, respectively, whereas the right handed Majorana neutrinos are unified into a $T_{7}$ triplet. Regarding the quark sector, we assign the quarks fields into trivial $T_{7}$ singlet representations. Note that SM right handed quarks are the only quark fields transforming non trivially under the $Z_{3}$ symmetry. Besides that, let's note that the right handed SM down type quarks are the only fermions odd under the $Z_{2}$ symmetry. Furthermore, it is worth mentioning that the $S U(3)_{L}$ scalar triplets are assigned to a $T_{7}$ trivial singlet representation, whereas the $S U(3)_{L}$ scalar singlets are accomodated into two $T_{7}$ triplets, one $T_{7}$ antitriplet, one $T_{7}$ trivial singlet and one $T_{7}$ non trivial singlet. The $S U(3)_{L}$ scalar singlets $T_{7}$ triplets are distinguished by their $Z_{3}$ charge assignments.

With the aforementioned field content of our model, the relevant quark and lepton Yukawa terms invariant under the group $\mathcal{G}$, take the form:

$$
\begin{aligned}
-\mathcal{L}_{Y}^{(Q)}= & y_{11}^{(U)} \bar{Q}_{L}^{1} \rho^{*} U_{R}^{1} \frac{\sigma^{4}}{\Lambda^{4}}+y_{12}^{(U)} \bar{Q}_{L}^{1} \rho^{*} U_{R}^{2} \frac{\sigma^{3}}{\Lambda^{3}}+y_{21}^{(U)} \bar{Q}_{L}^{2} \rho^{*} U_{R}^{1} \frac{\sigma^{3}}{\Lambda^{3}}+y_{22}^{(U)} \bar{Q}_{L}^{2} \rho^{*} U_{R}^{2} \frac{\sigma^{2}}{\Lambda^{2}} \\
& +y_{13}^{(U)} \bar{Q}_{L}^{1} \rho^{*} U_{R}^{3} \frac{\sigma^{2}}{\Lambda^{2}}+y_{31}^{(U)} \bar{Q}_{L}^{3} \eta U_{R}^{1} \frac{\sigma^{2}}{\Lambda^{2}}+y_{23}^{(U)} \bar{Q}_{L}^{2} \rho^{*} U_{R}^{3} \frac{\sigma}{\Lambda}+y_{32}^{(U)} \bar{Q}_{L}^{3} \eta U_{R}^{2} \frac{\sigma}{\Lambda} \\
& +y_{33}^{(U)} \bar{Q}_{L}^{3} \eta U_{R}^{3}+y^{(T)} \bar{Q}_{L}^{3} \chi T_{R}+y_{1}^{(J)} \bar{Q}_{L}^{1} \chi^{*} J_{R}^{1}+y_{2}^{(J)} \bar{Q}_{L}^{2} \chi^{*} J_{R}^{2}+y_{33}^{(D)} \bar{Q}_{L}^{3} \rho D_{R}^{3} \frac{\tau^{3}}{\Lambda^{3}} \\
& +y_{11}^{(D)} \bar{Q}_{L}^{1} \eta^{*} D_{R}^{1} \frac{\sigma^{4} \tau^{3}}{\Lambda^{7}}+y_{12}^{(D)} \bar{Q}_{L}^{1} \eta^{*} D_{R}^{2} \frac{\sigma^{3} \tau^{3}}{\Lambda^{6}}+y_{21}^{(D)} \bar{Q}_{L}^{2} \eta^{*} D_{R}^{1} \frac{\sigma^{3} \tau^{3}}{\Lambda^{6}}+y_{22}^{(D)} \bar{Q}_{L}^{2} \eta^{*} D_{R}^{2} \frac{\sigma^{2} \tau^{3}}{\Lambda^{5}} \\
& +y_{13}^{(D)} \bar{Q}_{L}^{1} \eta^{*} D_{R}^{3} \frac{\sigma^{2} \tau^{3}}{\Lambda^{5}}+y_{31}^{(D)} \bar{Q}_{L}^{3} \rho D_{R}^{1} \frac{\sigma^{2} \tau^{3}}{\Lambda^{5}}+y_{23}^{(D)} \bar{Q}_{L}^{2} \eta^{*} D_{R}^{3} \frac{\sigma \tau^{3}}{\Lambda}+y_{32}^{(D)} \bar{Q}_{L}^{3} \rho D_{R}^{2} \frac{\sigma \tau^{3}}{\Lambda}+H . c, \\
& -\mathcal{L}_{Y}^{(L)}=h_{\rho e}^{(L)}\left(\bar{L}_{L} \rho \xi\right)_{\mathbf{1}_{0}} e_{R} \frac{\sigma^{7}}{\Lambda^{8}}+h_{\rho \mu}^{(L)}\left(\bar{L}_{L} \rho \xi\right)_{\mathbf{1}_{2}} \mu_{R} \frac{\sigma^{4}}{\Lambda^{5}}+h_{\rho \tau}^{(L)}\left(\bar{L}_{L} \rho \xi\right)_{\mathbf{1}_{1}} \tau_{R} \frac{\sigma^{2}}{\Lambda^{3}} \\
& +h_{\chi}^{(L)}\left(\bar{L}_{L} \chi N_{R}\right)_{\mathbf{1}_{0}}+\frac{1}{2} h_{1 N}\left(\bar{N}_{R} N_{R}^{C}\right)_{\mathbf{3}} \xi+h_{2 N}\left(\bar{N}_{R} N_{R}^{C}\right)_{\overline{\mathbf{3}}} S \\
& +h_{\rho} \varepsilon_{a b c}\left(\bar{L}_{L}^{a}\left(L_{L}^{C}\right)^{b}\right)_{\mathbf{3}}\left(\rho^{*}\right)^{c} \frac{\zeta}{\Lambda}+H . c,
\end{aligned}
$$

where $y_{i j}^{(U, D)}(i, j=1,2,3), h_{\rho e}^{(L)}, h_{\rho \mu}^{(L)}, h_{\rho \tau}^{(L)}, h_{\chi}^{(L)}, h_{1 N}, h_{2 N}$ and $h_{\rho}$ are $\mathcal{O}(1)$ dimensionless couplings.

In the following we explain the role each discrete group factors of our model. The $T_{7}$ and $Z_{3}$ discrete groups reduce the number of the $S U(3)_{C} \otimes S U(3)_{L} \otimes U(1)_{X}$ model parameters. This allow us to get predictive and viable textures for the fermion sector that successfully describe the prevailing pattern of fermion masses and mixings, as we will show in sections [II and IV We use $T_{7}$ since it is the minimal non-Abelian discrete group having a complex triplet [10], where the three fermion generations can be naturally unified. The $Z_{3}$ symmetry determines the allowed entries of the neutrino mass matrix. Furthermore, the $Z_{3}$ symmetry distinguishes the right handed exotic quaks, 
being neutral under $Z_{3}$ from the right handed SM quarks, charged under this symmetry. This results in the absence of mixing between SM quarks and exotic quarks. Consequently, the $Z_{3}$ symmetry is crucial for decoupling the SM quarks from the exotic quarks. The $Z_{2}$ symmetry separates the right handed SM down type quarks, odd under this symmetry, from the SM up type quarks, even under $Z_{2}$. Consequently, the $Z_{2}$ symmetry is responsible for the mass hierarchy between SM up and SM down type quarks. Note that the $S U(3)_{L}$ scalar singlet $\tau$, the only $T_{7}$ non trivial singlet and $Z_{2}$ odd scalar, only appears in the SM down type quark Yukawa terms. The $Z_{14}$ symmetry generates the hierarchy among charged fermion masses and quark mixing angles that yields the observed charged fermion mass and quark mixing pattern. Furthermore, it is noteworthy that the five dimensional Yukawa operators $\frac{1}{\Lambda}\left(\bar{L}_{L} \rho \xi\right)_{\mathbf{1}_{0}} e_{R}, \frac{1}{\Lambda}\left(\bar{L}_{L} \rho \xi\right)_{\mathbf{1}_{2}} \mu_{R}$ and $\frac{1}{\Lambda}\left(\bar{L}_{L} \rho \xi\right)_{\mathbf{1}_{1}} \tau_{R}$ are invariant under $T_{7}$ but not under the $Z_{14}$ symmetry, because the right handed charged leptons are $Z_{14}$ charged. We use $Z_{14}$ because it is the smallest lowest cyclic symmetry, that allows to build a twelve dimensional charged lepton Yukawa term, from a $\frac{\sigma^{7}}{\Lambda^{7}}$ insertion on the $\frac{1}{\Lambda}\left(\bar{L}_{L} \rho \xi\right)_{\mathbf{1}_{0}} e_{R}$ operator. That aforementioned twelve dimensional charged lepton Yukawa term is crucial to explain the smallness of the electron mass, without tuning its corresponding Yukawa coupling.

To get a predictive model that successfully accounts for fermion masses and mixings, we assume that the $S U(3)_{L}$ singlet scalars have the following VEV pattern:

$$
\langle\sigma\rangle=v_{\sigma} e^{i \phi}, \quad\langle\tau\rangle=v_{\tau}, \quad\langle\xi\rangle=\frac{v_{\xi}}{\sqrt{3}}(1,1,1), \quad\langle\zeta\rangle=\frac{v_{\zeta}}{\sqrt{2}}(1,0,1), \quad\langle S\rangle=\frac{v_{S}}{\sqrt{3}}(1,1,-1) .
$$

We justify this choice of directions in the $T_{7}$ space by the observation that they describe a natural solution of the scalar potential minimization equations. Indeed, in the single-field case, $T_{7}$ invariance readily favors the $(1,1,1)$ direction over e.g. the $(1,0,0)$ solution for large regions of parameter space. The vacuum $\langle\xi\rangle$ is a configuration that preserves a $Z_{3}$ subgroup of $T_{7}$, which has been extensively studied by many authors, (see for example Ref [35]). In the next subsection, we consider the minimization conditions of the high energy scalar potential (17) of our model, and show that our chosen VEV directions for the two $T_{7}$ triplets, i.e., $\xi, S$ and the $T_{7}$ antitriplet $\zeta$ scalars in Eq. (14), are consistent with a global minimum of this scalar potential.

Besides that, the $S U(3)_{L}$ scalar singlets are assumed to acquire vacuum expectation values at a very high energy $\Lambda_{\text {int }} \gg v_{\chi} \approx \mathcal{O}(1) \mathrm{TeV}$, excepting $\zeta_{j}(j=1,2,3)$, whose vacuum expectation value is much lower than the scale of electroweak symmetry breaking $v=246 \mathrm{GeV}$. Let's note that at the scale $\Lambda_{\text {int }}$, the $S U(3)_{C} \otimes S U(3)_{L} \otimes U(1)_{X} \otimes T_{7} \otimes$ $Z_{2} \otimes Z_{3} \otimes Z_{14}$ symmetry is broken to $S U(3)_{C} \otimes S U(3)_{L} \otimes U(1)_{X}$ by the vacuum expectation values of the $S U(3)_{L}$ singlet scalar fields $\xi_{j}, S_{j}, \sigma$ and $\tau$.

Considering that the charged fermion mass and quark mixing pattern arises from the $Z_{2} \otimes Z_{3} \otimes Z_{14}$ symmetry breaking, we set the VEVs of the $S U(3)_{L}$ singlet scalars $S, \xi, \sigma$ and $\tau$, as follows:

$$
v_{S} \sim v_{\xi}=v_{\sigma}=v_{\tau}=\Lambda_{\text {int }}=\lambda \Lambda,
$$

being $\lambda=0.225$ one of the Wolfenstein parameters and $\Lambda$ our model cutoff. Consequently, the VEVs of the scalars in our model have the following hierarchy:

$$
v_{\zeta}<<v_{\rho} \sim v_{\eta} \sim v<<v_{\chi}<<\Lambda_{\text {int }} .
$$

Thus, the $S U(3)_{L}$ scalar singlets having Yukawa interactions with the right handed Majorana neutrinos get VEVs at very high scale, then providing very large masses to these Majorana neutrinos, and thus giving rise to a double seesaw mechanism of active neutrino masses. Consequently, the neutrino spectrum includes very light active neutrinos as well as heavy and very heavy sterile neutrinos. As we will shown in detail in the next section, the smallness of the active neutrino masses is attributed to their scaling with inverse powers of the high energy cutoff $\Lambda$ as well as by their quadratic dependence on the very small VEV of the $Z_{2} \otimes Z_{3} \otimes Z_{14}$ neutral, $S U(3)_{L}$ singlet and $T_{7}$ antitriplet scalar field $\zeta$.

\section{B. High energy scalar potential}

As previously mentioned, all singlet scalars, excepting $\zeta_{j}(j=1,2,3)$, acquire vaccum expectation values, much larger than $v_{\chi}$, which implies that the singlet scalars are very heavy and thus the mixing between these scalar singlets and the $S U(3)_{L}$ scalar triplets can be neglected as done in Ref. 30]. For simplicity we assume a CP invariant scalar potential with only real couplings as done in Refs. [15, 29, 30, 42]. The high energy scalar potential which involves only the $S U(3)_{L}$ singlet slcalars is given by: 


$$
\begin{aligned}
& V_{1}=\mu_{\xi}^{2}\left(\xi \xi^{*}\right)_{\mathbf{1}_{0}}+\mu_{S}^{2}\left(S S^{*}\right)_{\mathbf{1}_{0}}+\mu_{\zeta}^{2}\left(\zeta \zeta^{*}\right)_{\mathbf{1}_{0}}+\mu_{\sigma}^{2}\left(\sigma \sigma^{*}\right)+\mu_{\tau}^{2}\left(\tau \tau^{*}\right) \\
& +\left[C_{1}\left(\xi \xi^{*}\right)_{\mathbf{3}} \zeta+C_{2}\left(S S^{*}\right)_{\mathbf{3}} \zeta+C_{3}(\xi S)_{\mathbf{3}} \zeta+C_{4}(\xi \zeta)_{\mathbf{3}} \xi^{*}+C_{5}(S \zeta)_{\mathbf{3}} S^{*}+C_{6}\left(\xi^{*} \zeta\right)_{\overline{\mathbf{3}}} \xi\right. \\
& +C_{7}\left(S^{*} \zeta\right)_{\overline{3}} S+C_{8}(\xi \zeta)_{\overline{3}} S+C_{9}(S \zeta)_{\overline{3}} \xi+C_{10}(\zeta \zeta)_{\mathbf{3}} \zeta+C_{11}(\zeta \zeta)_{\overline{3}} \zeta^{*}+C_{10}\left(\zeta \zeta^{*}\right)_{\mathbf{3}} \zeta \\
& \left.+C_{15}\left(\zeta \zeta^{*}\right)_{\overline{\mathbf{3}}} \zeta^{*}+C_{12}(\xi S)_{\overline{\mathbf{3}}} \zeta^{*}+C_{13}\left(\xi^{*} \zeta\right)_{\mathbf{3}} S^{*}+C_{14}\left(S^{*} \zeta\right)_{\mathbf{3}} \xi^{*}+H . c\right]+\kappa_{1}\left(\xi \xi^{*}\right)_{\mathbf{1}_{0}}\left(\xi \xi^{*}\right)_{\mathbf{1}_{0}} \\
& +\kappa_{2}\left(\xi \xi^{*}\right)_{\mathbf{1}_{1}}\left(\xi \xi^{*}\right)_{\mathbf{1}_{2}}+\kappa_{3}\left(\xi \xi^{*}\right)_{\mathbf{3}}\left(\xi \xi^{*}\right)_{\overline{\mathbf{3}}}+\kappa_{4}\left(S S^{*}\right)_{\mathbf{1}_{0}}\left(S S^{*}\right)_{\mathbf{1}_{0}}+\kappa_{5}\left(S S^{*}\right)_{\mathbf{1}_{1}}\left(S S^{*}\right)_{\mathbf{1}_{2}} \\
& +\kappa_{6}\left(S S^{*}\right)_{\mathbf{3}}\left(S S^{*}\right)_{\overline{\mathbf{3}}}+\kappa_{7}\left(\zeta \zeta^{*}\right)_{\mathbf{1}_{0}}\left(\zeta \zeta^{*}\right)_{\mathbf{1}_{0}}+\kappa_{8}\left(\zeta \zeta^{*}\right)_{\mathbf{1}_{1}}\left(\zeta \zeta^{*}\right)_{\mathbf{1}_{2}}+\kappa_{9}\left(\zeta \zeta^{*}\right)_{\mathbf{3}}\left(\zeta \zeta^{*}\right)_{\overline{\mathbf{3}}} \\
& +\left[\kappa_{10}(\zeta \zeta)_{\mathbf{3}}(\zeta \zeta)_{\overline{\mathbf{3}}}+\gamma_{75}(\xi S)_{\mathbf{3}}(\xi S)_{\overline{\mathbf{3}}}+\gamma_{1}(\xi \xi)_{\mathbf{3}}(S S)_{\overline{\mathbf{3}}}+\gamma_{2}(S S)_{\mathbf{3}}(\xi \xi)_{\overline{\mathbf{3}}}+H . c\right] \\
& +\gamma_{3}\left(\xi \xi^{*}\right)_{\mathbf{1}_{0}}\left(S S^{*}\right)_{\mathbf{1}_{0}}+\gamma_{4}\left(\xi \xi^{*}\right)_{\mathbf{1}_{1}}\left(S S^{*}\right)_{\mathbf{1}_{2}}+\gamma_{5}\left(\xi \xi^{*}\right)_{\mathbf{1}_{2}}\left(S S^{*}\right)_{\mathbf{1}_{1}}+\gamma_{6}\left(\xi \xi^{*}\right)_{\mathbf{3}}\left(S S^{*}\right)_{\overline{\mathbf{3}}} \\
& +\gamma_{7}\left(\xi \xi^{*}\right)_{\overline{\mathbf{3}}}\left(S S^{*}\right)_{\mathbf{3}}+\left[\gamma_{14}(\zeta \zeta)_{\mathbf{3}}(S S)_{\overline{\mathbf{3}}}+\gamma_{15}(\zeta \zeta)_{\mathbf{3}}(\xi \xi)_{\overline{\mathbf{3}}}+H . c\right]+\gamma_{8}\left(\xi S^{*}\right)_{\mathbf{1}_{0}}\left(S \xi^{*}\right)_{\mathbf{1}_{0}} \\
& +\gamma_{9}\left(\xi S^{*}\right)_{\mathbf{1}_{1}}\left(S \xi^{*}\right)_{\mathbf{1}_{2}}+\gamma_{11}\left(\xi S^{*}\right)_{\mathbf{1}_{\mathbf{2}}}\left(S \xi^{*}\right)_{\mathbf{1}_{1}}+\gamma_{12}(\xi S)_{\mathbf{3}}\left(\xi^{*} S^{*}\right)_{\overline{\mathbf{3}}}+\gamma_{13}\left(\xi^{*} S^{*}\right)_{\mathbf{3}}(\xi S)_{\overline{\mathbf{3}}} \\
& +\gamma_{17}\left(\xi S^{*}\right)_{\mathbf{3}}\left(\xi^{*} S\right)_{\overline{\mathbf{3}}}+\gamma_{18}\left(\xi^{*} S\right)_{\mathbf{3}}\left(\xi S^{*}\right)_{\overline{\mathbf{3}}}+\gamma_{19}\left(\xi \xi^{*}\right)_{\mathbf{1}_{0}}\left(\zeta \zeta^{*}\right)_{\mathbf{1}_{0}}+\gamma_{20}\left(\xi \xi^{*}\right)_{\mathbf{1}_{1}}\left(\zeta \zeta^{*}\right)_{\mathbf{1}_{2}} \\
& +\gamma_{21}\left(\xi \xi^{*}\right)_{\mathbf{1}_{2}}\left(\zeta \zeta^{*}\right)_{\mathbf{1}_{1}}+\gamma_{23}\left(\xi \xi^{*}\right)_{\mathbf{3}}\left(\zeta \zeta^{*}\right)_{\overline{\mathbf{3}}}+\gamma_{24}\left(\zeta \zeta^{*}\right)_{\mathbf{3}}\left(\xi \xi^{*}\right)_{\overline{\mathbf{3}}} \\
& +\left[\gamma_{25}\left(\xi \xi^{*}\right)_{\mathbf{3}}(\zeta \zeta)_{\overline{\mathbf{3}}}+\gamma_{26}(\zeta \zeta)_{\mathbf{3}}\left(\xi \xi^{*}\right)_{\overline{\mathbf{3}}}+H . c\right]+\gamma_{30}(\xi \zeta)_{\mathbf{3}}\left(\xi^{*} \zeta^{*}\right)_{\overline{\mathbf{3}}} \\
& +\gamma_{27}(\xi \zeta)_{\mathbf{1}_{0}}\left(\xi^{*} \zeta^{*}\right)_{\mathbf{1}_{0}}+\gamma_{28}(\xi \zeta)_{\mathbf{1}_{1}}\left(\xi^{*} \zeta^{*}\right)_{\mathbf{1}_{2}}+\gamma_{29}(\xi \zeta)_{\mathbf{1}_{2}}\left(\xi^{*} \zeta^{*}\right)_{\mathbf{1}_{1}} \\
& +\gamma_{31}\left(\xi^{*} \zeta^{*}\right)_{\mathbf{3}}(\xi \zeta)_{\overline{\mathbf{3}}}+\left[\gamma_{32}(\xi \zeta)_{\mathbf{3}}\left(\xi^{*} \zeta\right)_{\overline{\mathbf{3}}}+\gamma_{33}\left(\xi^{*} \zeta\right)_{\mathbf{3}}(\xi \zeta)_{\overline{\mathbf{3}}}+H . c\right] \\
& +\gamma_{34}\left(S S^{*}\right)_{\mathbf{1}_{0}}\left(\zeta \zeta^{*}\right)_{\mathbf{1}_{0}}+\gamma_{35}\left(S S^{*}\right)_{\mathbf{1}_{1}}\left(\zeta \zeta^{*}\right)_{\mathbf{1}_{2}}+\gamma_{36}\left(S S^{*}\right)_{\mathbf{1}_{2}}\left(\zeta \zeta^{*}\right)_{\mathbf{1}_{1}}+\gamma_{37}\left(S S^{*}\right)_{\mathbf{3}}\left(\zeta \zeta^{*}\right)_{\overline{\mathbf{3}}} \\
& +\gamma_{38}\left(\zeta \zeta^{*}\right)_{\mathbf{3}}\left(S S^{*}\right)_{\overline{\mathbf{3}}}+\left[\gamma_{39}\left(S S^{*}\right)_{\mathbf{3}}(\zeta \zeta)_{\overline{\mathbf{3}}}+\gamma_{40}(\zeta \zeta)_{\mathbf{3}}\left(S S^{*}\right)_{\overline{\mathbf{3}}}+H . c\right]+\gamma_{44}(S \zeta)_{\mathbf{3}}\left(S^{*} \zeta^{*}\right)_{\overline{\mathbf{3}}} \\
& +\gamma_{41}(S \zeta)_{\mathbf{1}_{0}}\left(S^{*} \zeta^{*}\right)_{\mathbf{1}_{0}}+\gamma_{42}(S \zeta)_{\mathbf{1}_{1}}\left(S^{*} \zeta^{*}\right)_{\mathbf{1}_{2}}+\gamma_{43}(S \zeta)_{\mathbf{1}_{2}}\left(S^{*} \zeta^{*}\right)_{\mathbf{1}_{1}}+\gamma_{45}\left(S^{*} \zeta^{*}\right)_{\mathbf{3}}(S \zeta)_{\overline{\mathbf{3}}} \\
& +\left[\gamma_{49}(\xi S)_{\mathbf{3}}\left(\zeta \zeta^{*}\right)_{\overline{\mathbf{3}}}+\gamma_{50}\left(\zeta \zeta^{*}\right)_{\mathbf{3}}(\xi S)_{\overline{\mathbf{3}}}+\gamma_{51}(\xi S)_{\mathbf{3}}(\zeta \zeta)_{\overline{\mathbf{3}}}+\gamma_{52}(\zeta \zeta)_{\mathbf{3}}(\xi S)_{\overline{\mathbf{3}}}+\text { H.c }\right] \\
& +\left[\gamma_{53}(\xi \zeta)_{\mathbf{1}_{0}}(S \zeta)_{\mathbf{1}_{0}}+\gamma_{54}(\xi \zeta)_{\mathbf{1}_{1}}(S \zeta)_{\mathbf{1}_{2}}+\gamma_{55}(\xi \zeta)_{\mathbf{1}_{2}}(S \zeta)_{\mathbf{1}_{1}}\right. \\
& \left.+\gamma_{56}(\xi \zeta)_{\mathbf{3}}(S \zeta)_{\overline{\mathbf{3}}}+\gamma_{57}(S \zeta)_{\mathbf{3}}(\xi \zeta)_{\overline{\mathbf{3}}}+H . c\right]+\gamma_{66}\left(\zeta \zeta^{*}\right)_{\mathbf{1}_{0}}\left(\sigma \sigma^{*}\right)+\gamma_{16}\left(\zeta \zeta^{*}\right)_{\mathbf{1}_{0}}\left(\sigma \sigma^{*}\right) \\
& +\gamma_{58}\left(\xi \xi^{*}\right)_{\mathbf{1}_{0}}\left(\sigma \sigma^{*}\right)+\gamma_{59}\left(S S^{*}\right)_{\mathbf{1}_{0}}\left(\sigma \sigma^{*}\right)+\gamma_{16}\left(\zeta \zeta^{*}\right)_{\mathbf{1}_{1}} \zeta^{2}+\gamma_{67}\left(\zeta \zeta^{*}\right)_{\mathbf{1}_{2}} \zeta^{* 2} \\
& +\gamma_{61}\left(\xi \xi^{*}\right)_{\mathbf{1}_{0}}\left(\tau \tau^{*}\right)+\gamma_{62}\left(S S^{*}\right)_{\mathbf{1}_{0}}\left(\tau \tau^{*}\right)+\gamma_{68}\left(\sigma \sigma^{*}\right)^{2}+\gamma_{69}\left(\tau \tau^{*}\right)^{2}+\gamma_{70}\left(\sigma \sigma^{*}\right)\left(\tau \tau^{*}\right) \\
& +\gamma_{71}\left(\xi \xi^{*}\right)_{\mathbf{1}_{1}} \tau^{2}+\gamma_{72}\left(\xi \xi^{*}\right)_{\mathbf{1}_{2}} \tau^{* 2}+\gamma_{73}\left(S S^{*}\right)_{\mathbf{1}_{1}} \tau^{2}+\gamma_{74}\left(S S^{*}\right)_{\mathbf{1}_{2}} \tau^{* 2}
\end{aligned}
$$

Now we are going to determine the conditions under which the VEV pattern for the $S U(3)_{L}$ singlet scalars, given in Eq. (14), is a solution of the high energy scalar potential. In view of the very large number of parameters of the high energy scalar potential, in order to simplify the analysis, we assume universality in its trilinear and quartic couplings, i.e.

$$
\begin{array}{rlrl}
\kappa_{i} & =\gamma_{j}=\kappa, \quad & i=(1-10), & j=(1-75), \\
C_{k} & =C, & k=(1-15)
\end{array}
$$

Then, from the minimization conditions of the scalar potential and taking into account our assumption that $v_{\zeta_{j}}$ $(j=1,2,3)$ are much lower than the electroweak symmetry breaking scale $v=246 \mathrm{GeV}$, the following relations are 
obtained:

$$
\begin{aligned}
\frac{\partial\left\langle V_{1}\right\rangle}{\partial v_{\xi_{1}}}= & 8 \kappa v_{\xi_{1}}^{3}+4 \kappa v_{\xi_{2}}^{3}+2 v_{\xi_{1}} \mu_{\xi}^{2}+2 \kappa v_{\xi_{1}}\left(v_{\xi_{2}}^{2}+v_{\xi_{3}}^{2}\right)+12 \kappa v_{\xi_{1}}^{2} v_{\xi_{3}}+2 \kappa v_{\xi_{1}} v_{\xi_{2}}^{2}+2 \kappa v_{S_{1}}^{2}\left(7 v_{\xi_{1}}+3 v_{\xi_{3}}\right) \\
& +2 \kappa v_{S_{2}}^{2}\left(2 v_{\xi_{1}}+3 v_{\xi_{3}}\right)+4 \kappa v_{S_{3}}^{2} v_{\xi_{1}}+2 \kappa\left(v_{\sigma}^{2}+3 v_{\tau}^{2}\right) v_{\xi_{1}}+4 \kappa v_{S_{1}}\left(v_{\xi_{2}} v_{S_{2}}+v_{\xi_{3}} v_{S_{3}}+3 v_{\xi_{1}} v_{S_{3}}\right) \\
= & 0, \\
\frac{\partial\left\langle V_{1}\right\rangle}{\partial v_{\xi_{2}}}= & 8 \kappa v_{\xi_{2}}^{3}+4 \kappa v_{\xi_{3}}^{3}+2 v_{\xi_{2}} \mu_{\xi}^{2}+2 \kappa v_{\xi_{2}}\left(v_{\xi_{1}}^{2}+v_{\xi_{3}}^{2}\right)+12 \kappa v_{\xi_{2}}^{2} v_{\xi_{1}}+2 \kappa v_{\xi_{2}} v_{\xi_{3}}^{2}+2 \kappa v_{S_{2}}^{2}\left(7 v_{\xi_{2}}+3 v_{\xi_{1}}\right) \\
& +2 \kappa v_{S_{3}}^{2}\left(2 v_{\xi_{2}}+3 v_{\xi_{3}}\right)+4 \kappa v_{S_{1}}^{2} v_{\xi_{2}}+2 \kappa v_{\sigma}^{2} v_{\xi_{2}}+4 \kappa v_{S_{2}}\left(v_{\xi_{1}} v_{S_{1}}+v_{\xi_{3}} v_{S_{3}}+3 v_{\xi_{2}} v_{S_{1}}\right) \\
\frac{\partial\left\langle V_{1}\right\rangle}{\partial v_{\xi_{3}}}= & 8 \kappa v_{\xi_{3}}^{3}+4 \kappa v_{\xi_{1}}^{3}+2 v_{\xi_{3}} \mu_{\xi}^{2}+2 \kappa v_{\xi_{3}}\left(v_{\xi_{1}}^{2}+v_{\xi_{2}}^{2}\right)+12 \kappa v_{\xi_{3}}^{2} v_{\xi_{2}}+2 \kappa v_{\xi_{3}} v_{\xi_{2}}^{2}+2 \kappa v_{S_{3}}^{2}\left(7 v_{\xi_{3}}+3 v_{\xi_{2}}\right) \\
& +2 \kappa v_{S_{1}}^{2}\left(2 v_{\xi_{3}}+3 v_{\xi_{1}}\right)+4 \kappa v_{S_{2}}^{2} v_{\xi_{3}}+2 \kappa v_{\sigma}^{2} v_{\xi_{3}}+4 \kappa v_{S_{3}}\left(v_{\xi_{2}} v_{S_{2}}+v_{\xi_{1}} v_{S_{1}}+3 v_{\xi_{3}} v_{S_{2}}\right) \\
= & 0,
\end{aligned}
$$

$$
\begin{aligned}
\frac{\partial\left\langle V_{1}\right\rangle}{\partial v_{S_{1}}}= & 8 \kappa v_{S_{1}}^{3}+4 \kappa v_{S_{2}}^{3}+2 v_{S_{1}} \mu_{\xi}^{2}+2 \kappa v_{S_{1}}\left(v_{S_{2}}^{2}+v_{S_{3}}^{2}\right)+12 \kappa v_{S_{1}}^{2} v_{S_{3}}+2 \kappa v_{S_{1}} v_{S_{2}}^{2}+2 \kappa v_{\xi_{1}}^{2}\left(7 v_{S_{1}}+3 v_{S_{3}}\right) \\
& +2 \kappa v_{\xi_{2}}^{2}\left(2 v_{S_{1}}+3 v_{S_{3}}\right)+4 \kappa v_{\xi_{3}}^{2} v_{S_{1}}+2 \kappa\left(v_{\sigma}^{2}+3 v_{\tau}^{2}\right) v_{S_{1}}+4 \kappa v_{\xi_{1}}\left(v_{S_{2}} v_{\xi_{2}}+v_{S_{3}} v_{\xi_{3}}+3 v_{S_{1}} v_{\xi_{3}}\right) \\
= & 0 \\
\frac{\partial\left\langle V_{1}\right\rangle}{\partial v_{S_{2}}}= & 8 \kappa v_{S_{2}}^{3}+4 \kappa v_{S_{3}}^{3}+2 v_{S_{2}} \mu_{\xi}^{2}+2 \kappa v_{S_{2}}\left(v_{S_{1}}^{2}+v_{S_{3}}^{2}\right)+12 \kappa v_{S_{2}}^{2} v_{S_{1}}+2 \kappa v_{S_{2}} v_{S_{3}}^{2}+2 \kappa v_{\xi_{2}}^{2}\left(7 v_{S_{2}}+3 v_{S_{1}}\right) \\
& +2 \kappa v_{\xi_{3}}^{2}\left(2 v_{S_{2}}+3 v_{S_{3}}\right)+4 \kappa v_{\xi_{1}}^{2} v_{S_{2}}+2 \kappa v_{\sigma}^{2} v_{S_{2}}+4 \kappa v_{\xi_{2}}\left(v_{S_{1}} v_{\xi_{1}}+v_{S_{3}} v_{\xi_{3}}+3 v_{S_{2}} v_{\xi_{1}}\right) \\
\frac{\partial\left\langle V_{1}\right\rangle}{\partial v_{S_{3}}=} & 8 \kappa v_{S_{3}}^{3}+4 \kappa v_{S_{1}}^{3}+2 v_{S_{3}} \mu_{\xi}^{2}+2 \kappa v_{S_{3}}\left(v_{S_{1}}^{2}+v_{S_{2}}^{2}\right)+12 \kappa v_{S_{3}}^{2} v_{S_{2}}+2 \kappa v_{S_{3}} v_{S_{2}}^{2}+2 \kappa v_{\xi_{3}}^{2}\left(7 v_{S_{3}}+3 v_{S_{2}}\right) \\
& +2 \kappa v_{\xi_{1}}^{2}\left(2 v_{S_{3}}+3 v_{S_{1}}\right)+4 \kappa v_{\xi_{2}}^{2} v_{S_{3}}+2 \kappa v_{\sigma}^{2} v_{S_{3}}+4 \kappa v_{\xi_{3}}\left(v_{S_{2}} v_{\xi_{2}}+v_{S_{1}} v_{\xi_{1}}+3 v_{S_{3}} v_{\xi_{2}}\right) \\
= & 0,
\end{aligned}
$$

$$
\begin{gathered}
\frac{\partial\left\langle V_{1}\right\rangle}{\partial v_{\zeta_{1}}}=6 C\left[v_{\xi_{1}} v_{\xi_{2}}+v_{S_{1}} v_{S_{2}}+2\left(v_{\xi_{3}} v_{S_{2}}+v_{\xi_{2}} v_{S_{3}}\right)+v_{\xi_{3}} v_{S_{3}}\right]+2 \mu_{\zeta}^{2} v_{\zeta_{1}}=0, \\
\frac{\partial\left\langle V_{1}\right\rangle}{\partial v_{\zeta_{2}}}=6 C\left[v_{\xi_{2}} v_{\xi_{3}}+v_{S_{2}} v_{S_{3}}+2\left(v_{\xi_{3}} v_{S_{1}}+v_{\xi_{1}} v_{S_{3}}\right)+v_{\xi_{1}} v_{S_{1}}\right]+2 \mu_{\zeta}^{2} v_{\zeta_{2}}=0, \\
\frac{\partial\left\langle V_{1}\right\rangle}{\partial v_{\zeta_{3}}}=6 C\left[v_{\xi_{1}} v_{\xi_{3}}+v_{S_{1}} v_{S_{3}}+2\left(v_{\xi_{2}} v_{S_{1}}+v_{\xi_{1}} v_{S_{2}}\right)+v_{\xi_{2}} v_{S_{2}}\right]+2 \mu_{\zeta}^{2} v_{\zeta_{3}}=0, \\
\frac{\partial\left\langle V_{1}\right\rangle}{\partial v_{\sigma}}=2 v_{\sigma}\left[\mu_{\sigma}^{2}+\kappa\left(v_{\xi_{1}}^{2}+v_{\xi_{2}}^{2}+v_{\xi_{3}}^{2}+v_{S_{1}}^{2}+v_{S_{2}}^{2}+v_{S_{3}}^{2}+2 v_{\sigma}^{2}+v_{\tau}^{2}\right)\right]=0, \\
\frac{\partial\left\langle V_{1}\right\rangle}{\partial v_{\tau}}=2 v_{\tau}\left[\mu_{\tau}^{2}+\kappa\left(3 v_{\xi_{1}}^{2}+3 v_{S_{1}}^{2}+v_{\sigma}^{2}+2 v_{\tau}^{2}\right)\right]=0 .
\end{gathered}
$$

From the expressions given above, and using the vacuum configuration for the $S U(3)_{L}$ singlet scalars given in Eq. (14), we find the following relations:

$$
\begin{array}{rlrl}
\mu_{\xi}^{2}= & -\frac{1}{9} \kappa\left(60 v_{\xi}^{2}+41 v_{S}^{2}\right), & \mu_{S}^{2}=-\frac{1}{9} \kappa\left(59 v_{\xi}^{2}+10 v_{S}^{2}\right), \\
\mu_{\zeta}^{2}=-\frac{\sqrt{2} C v_{\xi}}{v_{\zeta}}\left(v_{\xi}+v_{S}\right), & \mu_{\sigma}^{2}=\mu_{\tau}^{2}=-\kappa\left(4 v_{\xi}^{2}+v_{S}^{2}\right), \\
2 v_{\xi}^{2}-2 v_{S}^{2}+v_{\xi} v_{S}=0 . &
\end{array}
$$


Taking the positive solution of the previous equation, we find:

$$
v_{S}=\frac{1+\sqrt{5}}{4} v_{\xi} \simeq 0.81 v_{\xi}
$$

which is consistent with our previous assumption described by Eq. (15). Our results show that the VEV directions for the two $T_{7}$ triplets, i.e., $\xi, S$ and the $T_{7}$ antitriplet $\zeta$ scalars in Eq. (14), are consistent with a global minimum of the high scalar potential (17) of our model, for a not fine-tuned region of parameter space.

\section{Low energy scalar potential}

The renormalizable low energy scalar potential of the model takes the form:

$$
\begin{aligned}
& V_{H}=\mu_{\chi}^{2}\left(\chi^{\dagger} \chi\right)+\mu_{\eta}^{2}\left(\eta^{\dagger} \eta\right)+\mu_{\rho}^{2}\left(\rho^{\dagger} \rho\right)+f\left(\chi_{i} \eta_{j} \rho_{k} \varepsilon^{i j k}+H . c .\right)+\lambda_{1}\left(\chi^{\dagger} \chi\right)\left(\chi^{\dagger} \chi\right) \\
& +\lambda_{2}\left(\rho^{\dagger} \rho\right)\left(\rho^{\dagger} \rho\right)+\lambda_{3}\left(\eta^{\dagger} \eta\right)\left(\eta^{\dagger} \eta\right)+\lambda_{4}\left(\chi^{\dagger} \chi\right)\left(\rho^{\dagger} \rho\right)+\lambda_{5}\left(\chi^{\dagger} \chi\right)\left(\eta^{\dagger} \eta\right) \\
& +\lambda_{6}\left(\rho^{\dagger} \rho\right)\left(\eta^{\dagger} \eta\right)+\lambda_{7}\left(\chi^{\dagger} \eta\right)\left(\eta^{\dagger} \chi\right)+\lambda_{8}\left(\chi^{\dagger} \rho\right)\left(\rho^{\dagger} \chi\right)+\lambda_{9}\left(\rho^{\dagger} \eta\right)\left(\eta^{\dagger} \rho\right) .
\end{aligned}
$$

After the symmetry breaking, it is found that the scalar mass eigenstates are connected with the weak scalar states by the following relations: [14, 15]:

$$
\begin{gathered}
\left(\begin{array}{c}
G_{1}^{ \pm} \\
H_{1}^{ \pm}
\end{array}\right)=R_{\beta_{T}}\left(\begin{array}{c}
\rho_{1}^{ \pm} \\
\eta_{2}^{ \pm}
\end{array}\right), \quad\left(\begin{array}{c}
G_{1}^{0} \\
A_{1}^{0}
\end{array}\right)=R_{\beta_{T}}\left(\begin{array}{l}
\zeta_{\rho} \\
\zeta_{\eta}
\end{array}\right), \quad\left(\begin{array}{c}
H_{1}^{0} \\
h^{0}
\end{array}\right)=R_{\alpha_{T}}\left(\begin{array}{l}
\xi_{\rho} \\
\xi_{\eta}
\end{array}\right), \\
\left(\begin{array}{c}
G_{2}^{0} \\
H_{2}^{0}
\end{array}\right)=R\left(\begin{array}{l}
\chi_{1}^{0} \\
\eta_{3}^{0}
\end{array}\right), \quad\left(\begin{array}{c}
G_{3}^{0} \\
H_{3}^{0}
\end{array}\right)=R\left(\begin{array}{l}
\zeta_{\chi} \\
\xi_{\chi}
\end{array}\right), \quad\left(\begin{array}{c}
G_{2}^{ \pm} \\
H_{2}^{ \pm}
\end{array}\right)=R\left(\begin{array}{c}
\chi_{2}^{ \pm} \\
\rho_{3}^{ \pm}
\end{array}\right),
\end{gathered}
$$

with

$$
R_{\alpha_{T}\left(\beta_{T}\right)}=\left(\begin{array}{cc}
\cos \alpha_{T}\left(\beta_{T}\right) & \sin \alpha_{T}\left(\beta_{T}\right) \\
-\sin \alpha_{T}\left(\beta_{T}\right) & \cos \alpha_{T}\left(\beta_{T}\right)
\end{array}\right), \quad R=\left(\begin{array}{cc}
-1 & 0 \\
0 & 1
\end{array}\right)
$$

where $\tan \beta_{T}=v_{\eta} / v_{\rho}$, and $\tan 2 \alpha_{T}=M_{1} /\left(M_{2}-M_{3}\right)$ with:

$$
\begin{aligned}
& M_{1}=4 \lambda_{6} v_{\eta} v_{\rho}+2 \sqrt{2} f v_{\chi}, \\
& M_{2}=4 \lambda_{2} v_{\rho}^{2}-\sqrt{2} f v_{\chi} \tan \beta_{T}, \\
& M_{3}=4 \lambda_{3} v_{\eta}^{2}-\sqrt{2} f v_{\chi} / \tan \beta_{T} .
\end{aligned}
$$

The low energy physical scalar spectrum of our model includes: 4 massive charged Higgs $\left(H_{1}^{ \pm}, H_{2}^{ \pm}\right)$, one CP-odd Higgs $\left(A_{1}^{0}\right), 3$ neutral CP-even Higgs $\left(h^{0}, H_{1}^{0}, H_{3}^{0}\right)$ and 2 neutral Higgs $\left(H_{2}^{0}, \bar{H}_{2}^{0}\right)$ bosons. The scalar $h^{0}$ is identified with the SM-like $126 \mathrm{GeV}$ Higgs boson found at the LHC. It it noteworthy that the neutral Goldstone bosons $G_{1}^{0}, G_{3}^{0}, G_{2}^{0}$, $\bar{G}_{2}^{0}$ are associated to the longitudinal components of the $Z, Z^{\prime}, K^{0}$ and $\bar{K}^{0}$ gauge bosons, respectively. Furthermore, the charged Goldstone bosons $G_{1}^{ \pm}$and $G_{2}^{ \pm}$are associated to the longitudinal components of the $W^{ \pm}$and $K^{ \pm}$gauge bosons, respectively [12, 15].

\section{LEPTON MASSES AND MIXINGS}

From Eqs. (13), (14), (15) and using the product rules of the $T_{7}$ group given in Appendix $\AA$ it follows that the mass matrix for charged leptons is:

$$
M_{l}=V_{l L}^{\dagger} P_{l} \operatorname{diag}\left(m_{e}, m_{\mu}, m_{\tau}\right), \quad V_{l L}=\frac{1}{\sqrt{3}}\left(\begin{array}{ccc}
1 & 1 & 1 \\
1 & \omega & \omega^{2} \\
1 & \omega^{2} & \omega
\end{array}\right), \quad P_{l}=\left(\begin{array}{ccc}
e^{7 i \phi} & 0 & 0 \\
0 & e^{4 i \phi} & 0 \\
0 & 0 & e^{2 i \phi}
\end{array}\right), \quad \omega=e^{\frac{2 \pi i}{3}},
$$


where the charged lepton masses read:

$$
m_{e}=h_{\rho e}^{(L)} \lambda^{8} \frac{v_{\rho}}{\sqrt{2}}, \quad m_{\mu}=h_{\rho \mu}^{(L)} \lambda^{5} \frac{v_{\rho}}{\sqrt{2}}, \quad m_{\tau}=h_{\rho \tau}^{(L)} \lambda^{3} \frac{v_{\rho}}{\sqrt{2}} .
$$

Taking into account that $v_{\rho} \approx v=246 \mathrm{GeV}$, it follows that the charged lepton masses are related with the electroweak symmetry breaking scale by their scalings with powers of the Wolfenstein parameter $\lambda=0.225$, with $\mathcal{O}(1)$ coefficients.

In the concerning to the neutrino sector, the following neutrino mass terms arise:

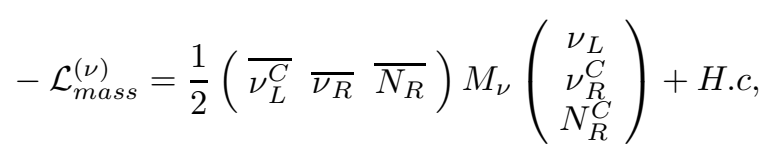

where the $T_{7}$ discrete flavor group constrains the neutrino mass matrix to be of the form:

$$
\begin{aligned}
& M_{\nu}=\left(\begin{array}{ccc}
0_{3 \times 3} & M_{D} & 0_{3 \times 3} \\
M_{D}^{T} & 0_{3 \times 3} & M_{\chi} \\
0_{3 \times 3} & M_{\chi}^{T} & M_{R}
\end{array}\right), \quad M_{D}=\frac{h_{\rho} v_{\rho} v_{\zeta}}{2 \Lambda}\left(\begin{array}{ccc}
0 & 1 & 0 \\
-1 & 0 & -1 \\
0 & 1 & 0
\end{array}\right), \quad M_{\chi}=h_{\chi}^{(L)} \frac{v_{\chi}}{\sqrt{2}}\left(\begin{array}{ccc}
1 & 0 & 0 \\
0 & 1 & 0 \\
0 & 0 & 1
\end{array}\right), \\
& M_{R}=h_{1 N} \frac{v_{\xi}}{\sqrt{3}}\left(\begin{array}{ccc}
1 & -x & x \\
-x & 1 & x \\
x & x & 1
\end{array}\right), x=\frac{h_{2 N} v_{S}}{h_{1 N} v_{\xi}} .
\end{aligned}
$$

Since the $S U(3)_{L}$ singlet scalars having Yukawa interactions with the right handed Majorana neutrinos acquire VEVs at very high scale, these Majorana neutrinos are very heavy, so that the active neutrinos get small masses via a double seesaw mechanism.

The full rotation matrix, which diagonalizes the neutrino mass matrix, takes the approximate form [41]:

$$
\mathbb{U}=\left(\begin{array}{ccc}
V_{\nu} & B_{2} U_{\chi} & 0 \\
-B_{2}^{\dagger} V_{\nu} & U_{\chi} & B_{1} U_{R} \\
0 & B_{1}^{\dagger} U_{\chi} & U_{R}
\end{array}\right)
$$

where

$$
B_{1}^{\dagger}=M_{R}^{-1} M_{\chi}^{T}, \quad B_{2}^{\dagger}=M_{D}\left(M_{\chi}^{T}\right)^{-1} M_{R} M_{\chi}^{-1},
$$

and the neutrino mass matrices for the physical states are:

$$
\begin{aligned}
& M_{\nu}^{(1)}=M_{D}\left(M_{\chi}^{T}\right)^{-1} M_{R} M_{\chi}^{-1} M_{D}^{T}, \\
& M_{\nu}^{(2)}=-M_{\chi} M_{R}^{-1} M_{\chi}^{T}, \\
& M_{\nu}^{(3)}=M_{R},
\end{aligned}
$$

being $M_{\nu}^{(1)}$ the mass matrix for light active neutrinos, while $M_{\nu}^{(2)}$ and $M_{\nu}^{(3)}$ are the heavy and very heavy sterile neutrino mass matrices, respectively. Consequently, the double seesaw mechanism gives rise to light active neutrinos as well as to heavy and very heavy sterile neutrinos. Moreover, the neutrino mass matrices $M_{\nu}^{(1)}, M_{\nu}^{(2)}$ and $M_{\nu}^{(3)}$ are diagonalized by the rotation matrices $V_{\nu}, U_{R}$ and $U_{\chi}$, respectively. [41].

Using Eq. (36), we find the following mass matrix for light active neutrinos:

$$
M_{\nu}^{(1)}=\left(\begin{array}{ccc}
A & 0 & A \\
0 & B & 0 \\
A & 0 & A
\end{array}\right)
$$

where

$$
A=\frac{h_{1 N} h_{\rho}^{2} v_{\rho}^{2} v_{\zeta}^{2} v_{\xi}}{2 \sqrt{3} h_{\chi}^{(L)} v_{\chi}^{2} \Lambda^{2}}, \quad B=\frac{h_{\rho}^{2} v_{\rho}^{2} v_{\zeta}^{2}}{\sqrt{3} h_{\chi}^{(L)} v_{\chi}^{2} \Lambda^{2}}\left(h_{1 N} v_{\xi}+h_{2 N} v_{S}\right)
$$

From Eq. (39) it follows that the light active neutrino mass matrix only depends on two effective parameters: $A$ and $B$, which determine the neutrino mass squared splittings. Let's note that $A$ and $B$ are supressed by their scaling with 
inverse powers of the high energy cutoff $\Lambda$. Furthermore, we have that the smallness of the active neutrino masses arises from their scaling with inverse powers of the high energy cutoff $\Lambda$ as well as from their quadratic dependence on the very small VEV of the $Z_{2} \otimes Z_{3} \otimes Z_{14}$ neutral, $S U(3)_{L}$ singlet and $T_{7}$ antitriplet scalar field $\zeta$. Considering that the orders of magnitude of the SM particles and new physics yield the constraints $v_{\chi} \gtrsim 1 \mathrm{TeV}$ and $v_{\eta}^{2}+v_{\rho}^{2}=v^{2}$ and taking into account our assumption that the dimensionless lepton Yukawa couplings are $\mathcal{O}(1)$ parameters, from Eq. (39) and the relations $v_{\xi}=\lambda \Lambda, v_{\rho} \sim 100 \mathrm{GeV}, v_{\chi} \sim 1 \mathrm{TeV}$, we get that the mass scale for the light active neutrinos satisfies $m_{\nu} \sim 10^{-3} \frac{v_{\zeta}^{2}}{\Lambda}$. Consequently, setting $v_{\zeta}=1 \mathrm{GeV}$, we find for the cutoff of our model the estimate

$$
\Lambda \sim 10^{5} \mathrm{TeV},
$$

which is of the same order of magnitude of the cutoff of our $S_{3}$ lepton flavor 331 model [29]. Consequenty, we find that the heavy and very heavy sterile neutrinos have masses at the $\sim \mathrm{MeV}$ and $\sim \mathrm{TeV}$ scales, respectively. Furthermore, from the aforementioned considerations, as well as from Eqs (15), (35) and (41), it follows that:

$$
\left|\left(B_{2}\right)_{i j}\right| \sim \frac{v_{\rho} v_{\zeta} v_{\xi}}{v_{\chi}^{2} \Lambda} \sim 10^{-5}<<\left|\left(B_{1}\right)_{i j}\right| \sim \frac{v_{\chi}}{v_{\xi}} \sim 10^{-3}, \quad i, j=1,2,3,
$$

Moreover, we find that the mass matrix $M_{\nu}^{(1)}$ for light active neutrinos is diagonalized by a rotation matrix $V_{\nu}$, as follows:

$$
V_{\nu}^{T} M_{\nu}^{(1)} V_{\nu}=\left(\begin{array}{ccc}
m_{1} & 0 & 0 \\
0 & m_{2} & 0 \\
0 & 0 & m_{3}
\end{array}\right), \quad \text { with } \quad V_{\nu}=\left(\begin{array}{ccc}
\cos \theta & 0 & \sin \theta \\
0 & 1 & 0 \\
-\sin \theta & 0 & \cos \theta
\end{array}\right), \quad \theta= \pm \frac{\pi}{4},
$$

where $\theta=+\pi / 4$ and $\theta=-\pi / 4$ correspond to normal (NH) and inverted (IH) mass hierarchies, respectively. The masses for the light active neutrinos, in the cases of normal $(\mathrm{NH})$ and inverted $(\mathrm{IH})$ mass hierarchies, read:

$$
\begin{array}{llll}
\mathrm{NH}: \theta=+\frac{\pi}{4}: & m_{\nu_{1}}=0, & m_{\nu_{2}}=B, & m_{\nu_{3}}=2 A, \\
\mathrm{IH}: \theta=-\frac{\pi}{4}: & m_{\nu_{1}}=2 A, & m_{\nu_{2}}=B, & m_{\nu_{3}}=0 .
\end{array}
$$

Besides that, the Pontecorvo-Maki-Nakagawa-Sakata (PMNS) leptonic mixing matrix has the following form:

$$
U=V_{l L}^{\dagger} P_{l} V_{\nu}=\left(\begin{array}{ccc}
\frac{e^{7 i \phi} \cos \theta}{\sqrt{3}}-\frac{e^{2 i \phi} \sin \theta}{\sqrt{3}} & \frac{e^{4 i \phi}}{\sqrt{3}} & \frac{e^{2 i \phi} \cos \theta}{\sqrt{3}}+\frac{e^{7 i \phi} \sin \theta}{\sqrt{3}} \\
\frac{e^{7 i \phi} \cos \theta}{\sqrt{3}}-\frac{e^{2 i \phi+\frac{2 i \pi}{3}} \sin \theta}{\sqrt{3}} & \frac{e^{4 i \phi-\frac{2 i \pi}{3}}}{\sqrt{3}} & \frac{e^{2 i \phi+\frac{2 i \pi}{3}} \cos \theta}{\sqrt{3}}+\frac{e^{7 i \phi} \sin \theta}{\sqrt{3}} \\
\frac{e^{7 i \phi} \cos \theta}{\sqrt{3}}-\frac{e^{2 i \phi-\frac{2 i \pi}{3}} \sin \theta}{\sqrt{3}} & \frac{e^{4 i \phi+\frac{2 i \pi}{3}}}{\sqrt{3}} & \frac{e^{2 i \phi-\frac{2 i \pi}{3}} \cos \theta}{\sqrt{3}}+\frac{e^{7 i \phi} \sin \theta}{\sqrt{3}}
\end{array}\right) .
$$

Note that while the PMNS leptonic mixing matrix only depends on a single parameter $\phi$ (which arises from the charged lepton mass matrix), the neutrino mass squared splittings are determined by two parameters, i.e., $A$ and $B$.

Furthermore, we find that the lepton mixing angles are given by:

$$
\begin{aligned}
& \sin ^{2} \theta_{12}=\frac{\left|U_{e 2}\right|^{2}}{1-\left|U_{e 3}\right|^{2}}=\frac{1}{2 \mp \cos 5 \phi}, \\
& \sin ^{2} \theta_{13}=\left|U_{e 3}\right|^{2}=\frac{1}{3}(1 \pm \cos 5 \phi), \\
& \sin ^{2} \theta_{23}=\frac{\left|U_{\mu 3}\right|^{2}}{1-\left|U_{e 3}\right|^{2}}=\frac{1}{2} \pm \frac{\sqrt{3} \sin 5 \phi)}{4 \mp 2 \cos 5 \phi} .
\end{aligned}
$$

Then, from Eq. (46), it follows that the limit $\phi=0$ and $\phi=\pi$ for the inverted and normal neutrino mass hierarchies, respectively, correspond to the trimaximal mixing, which predicts a vanishing reactor mixing angle. Let's note that the mixing angles for the lepton sector only depend on a single parameter $(\phi)$, while the neutrino mass squared splittings are controlled by two parameters, i.e., $A$ and $B$. 
The Jarlskog invariant and the $\mathrm{CP}$ violating phase are given by [6]:

$$
J=\operatorname{Im}\left(U_{e 1} U_{\mu 2} U_{e 2}^{*} U_{\mu 1}^{*}\right)=-\frac{1}{6 \sqrt{3}} \cos 2 \theta, \quad \sin \delta=\frac{8 J}{\cos \theta_{13} \sin 2 \theta_{12} \sin 2 \theta_{23} \sin 2 \theta_{13}} .
$$

From the relation $\theta= \pm \frac{\pi}{4}$, we predict $J=0$ and $\delta=0$, which implies a vanishing leptonic Dirac CP violating phase.

In the following the three free effective parameters $\phi, A$ and $B$ of the SM lepton sector of our model are adjusted to accommodate the experimental values of three leptonic mixing parameters and two neutrino mass squared splittings, shown in Tables I. II, for the normal (NH) and inverted (IH) neutrino mass hierarchies, respectively. The parameter $\phi$ is adjusted to reproduce the experimental values of the leptonic mixing parameters $\sin ^{2} \theta_{i j}$, whereas $A$ and $B$ for the normal $(\mathrm{NH})$ and inverted $(\mathrm{IH})$ neutrino mass hierarchies read:

$$
\begin{aligned}
& \mathrm{NH}: m_{\nu_{1}}=0, \quad m_{\nu_{2}}=B=\sqrt{\Delta m_{21}^{2}} \approx 9 \mathrm{meV}, \quad m_{\nu_{3}}=2 A=\sqrt{\Delta m_{31}^{2}} \approx 50 \mathrm{meV} ; \\
& \mathrm{IH}: m_{\nu_{2}}=B=\sqrt{\Delta m_{21}^{2}+\Delta m_{13}^{2}} \approx 50 \mathrm{meV}, \quad m_{\nu_{1}}=2 A=\sqrt{\Delta m_{13}^{2}} \approx 49 \mathrm{meV}, \quad m_{\nu_{3}}=0,
\end{aligned}
$$

which follows from Eqs. (45), (44) and the definition $\Delta m_{i j}^{2}=m_{i}^{2}-m_{j}^{2}$. We take the best fit values of $\Delta m_{i j}^{2}$ from Tables \and $\Pi$ for the normal and inverted neutrino mass hierarchies, respectively.

To reproduce the experimental values of the leptonic mixing parameters $\sin ^{2} \theta_{i j}$ given in Tables 【 W we vary the $\phi$ parameter, finding the following result:

$$
\begin{aligned}
& \mathrm{NH}: \phi=0.576 \pi, \quad \sin ^{2} \theta_{12} \approx 0.34, \quad \sin ^{2} \theta_{23} \approx 0.61, \quad \sin ^{2} \theta_{13} \approx 0.0232 \\
& \mathrm{IH}: \phi=0.376 \pi, \quad \sin ^{2} \theta_{12} \approx 0.34, \quad \sin ^{2} \theta_{23} \approx 0.61, \quad \sin ^{2} \theta_{13} \approx 0.0238 .
\end{aligned}
$$

Consequently, we find that $\sin ^{2} \theta_{13}$ is in excellent agreement with the experimental data, for both normal and inverted neutrino mass hierarchies, whereas $\sin ^{2} \theta_{12}$ and $\sin ^{2} \theta_{23}$ stay in the experimentally allowed $2 \sigma$ range. Thus, our predictions for the neutrino mass squared splittings and leptonic mixing parameters, are in very good agreement with the experimental data on neutrino oscillations, for both normal and inverted mass hierarchies. Furthermore, another relevant prediction of our model is a vanishing leptonic Dirac CP violating phase.

\begin{tabular}{|c|c|c|c|c|c|}
\hline Parameter & $\Delta m_{21}^{2}\left(10^{-5} \mathrm{eV}^{2}\right)$ & $\Delta m_{31}^{2}\left(10^{-3} \mathrm{eV}^{2}\right)$ & $\left(\sin ^{2} \theta_{12}\right)_{\exp }$ & $\left(\sin ^{2} \theta_{23}\right)_{\exp }$ & $\left(\sin ^{2} \theta_{13}\right)_{\exp }$ \\
\hline Best fit & 7.60 & 2.48 & 0.323 & 0.567 & 0.0234 \\
\hline $1 \sigma$ range & $7.42-7.79$ & $2.41-2.53$ & $0.307-0.339$ & $0.439-0.599$ & $0.0214-0.0254$ \\
\hline $2 \sigma$ range & $7.26-7.99$ & $2.35-2.59$ & $0.292-0.357$ & $0.413-0.623$ & $0.0195-0.0274$ \\
\hline $3 \sigma$ range & $7.11-8.11$ & $2.30-2.65$ & $0.278-0.375$ & $0.392-0.643$ & $0.0183-0.0297$ \\
\hline
\end{tabular}

Table I: Range for experimental values of neutrino mass squared splittings and leptonic mixing parameters, taken from Ref. [25], for the case of normal hierarchy.

\begin{tabular}{|c|c|c|c|c|c|}
\hline Parameter & $\Delta m_{21}^{2}\left(10^{-5} \mathrm{eV}^{2}\right)$ & $\Delta m_{13}^{2}\left(10^{-3} \mathrm{eV}^{2}\right)$ & $\left(\sin ^{2} \theta_{12}\right)_{\exp }$ & $\left(\sin ^{2} \theta_{23}\right)_{\exp }$ & $\left(\sin ^{2} \theta_{13}\right)_{\exp }$ \\
\hline Best fit & 7.60 & 2.38 & 0.323 & 0.573 & 0.0240 \\
\hline $1 \sigma$ range & $7.42-7.79$ & $2.32-2.43$ & $0.307-0.339$ & $0.530-0.598$ & $0.0221-0.0259$ \\
\hline $2 \sigma$ range & $7.26-7.99$ & $2.26-2.48$ & $0.292-0.357$ & $0.432-0.621$ & $0.0202-0.0278$ \\
\hline $3 \sigma$ range & $7.11-8.11$ & $2.20-2.54$ & $0.278-0.375$ & $0.403-0.640$ & $0.0183-0.0297$ \\
\hline
\end{tabular}

Table II: Range for experimental values of neutrino mass squared splittings and leptonic mixing parameters, taken from Ref. [25], for the case of inverted hierarchy.

Now we determine the effective Majorana neutrino mass parameter, which is proportional to the neutrinoless double beta $(0 \nu \beta \beta)$ decay amplitude. The effective Majorana neutrino mass parameter is given by:

$$
m_{\beta \beta}=\left|\sum_{j} U_{e k}^{2} m_{\nu_{k}}\right|,
$$


where $U_{e j}^{2}$ and $m_{\nu_{k}}$ are the PMNS mixing matrix elements and the Majorana neutrino masses, respectively.

From Eqs. (46), (51), (52) and (55), we find that that the effective Majorana neutrino mass parameter, for both Normal and Inverted hierarchies, takes the following values:

$$
m_{\beta \beta}=\left\{\begin{array}{lcr}
3 \mathrm{meV} & \text { for } & \mathrm{NH} \\
40 \mathrm{meV} & \text { for } & \mathrm{IH}
\end{array}\right.
$$

Our obtained values $m_{\beta \beta} \approx 3 \mathrm{meV}$ and $m_{\beta \beta} \approx 40 \mathrm{meV}$ for the effective Majorana neutrino mass parameter, for normal and inverted hierarchies, respectively, are beyond the reach of the present and forthcoming $0 \nu \beta \beta$ decay experiments. The current best upper bound on the effective neutrino mass is $m_{\beta \beta} \leq 160 \mathrm{meV}$, which corresponds to $T_{1 / 2}^{0 \nu \beta \beta}\left({ }^{136} \mathrm{Xe}\right) \geq$ $1.6 \times 10^{25}$ yr at $90 \%$ C.L, as indicated by the EXO-200 experiment [44]. This bound will be improved within a not too far future. The GERDA "phase-II" experiment [45, 46] is expected to reach $T_{1 / 2}^{0 \nu \beta \beta}\left({ }^{76} \mathrm{Ge}\right) \geq 2 \times 10^{26} \mathrm{yr}$, which corresponds to $m_{\beta \beta} \leq 100 \mathrm{meV}$. A bolometric CUORE experiment, using ${ }^{130} \mathrm{Te}$ [47], is currently under construction and has an estimated sensitivity of about $T_{1 / 2}^{0 \nu \beta \beta}\left({ }^{130} \mathrm{Te}\right) \sim 10^{26} \mathrm{yr}$, which corresponds to $m_{\beta \beta} \leq 50$ meV. Furthermore, there are proposals for ton-scale next-to-next generation $0 \nu \beta \beta$ experiments with ${ }^{136} \mathrm{Xe}$ [48, 49] and ${ }^{76} \mathrm{Ge}$ [45, 50] claiming sensitivities over $T_{1 / 2}^{0 \nu \beta \beta} \sim 10^{27} \mathrm{yr}$, which corresponds to $m_{\beta \beta} \sim 12-30 \mathrm{meV}$. For a recent review, see for example Ref. [51]. Consequently, as follows from Eq. (56), our model predicts $T_{1 / 2}^{0 \nu \beta \beta}$ at the level of sensitivities of the next generation or next-to-next generation $0 \nu \beta \beta$ experiments.

Regarding the sterile neutrino sector, from Eqs. (37) and (38) we find that the sterile neutrino mass matrices are given by:

$$
\begin{aligned}
& M_{\nu}^{(2)}=-\frac{\sqrt{3}\left(h_{\chi}^{(L)}\right)^{2} v_{\chi}^{2}}{2 h_{1 N} v_{\xi}}\left(\begin{array}{ccc}
X & -Y & Y \\
-Y & X & Y \\
Y & Y & X
\end{array}\right), \quad X=\frac{x-1}{2 x^{2}+x-1}, \quad X=\frac{x}{2 x^{2}+x-1} \\
& M_{\nu}^{(3)}=h_{1 N} \frac{v_{\xi}}{\sqrt{3}}\left(\begin{array}{ccc}
1 & -x & x \\
-x & 1 & x \\
x & x & 1
\end{array}\right), \quad x=\frac{h_{2 N} v_{S}}{h_{1 N} v_{\xi}}
\end{aligned}
$$

The sterile neutrino mass matrices $M_{\nu}^{(2)}$ and $M_{\nu}^{(3)}$ are diagonalized by a rotation matrix $U_{R}=U_{\chi}$, according to:

$$
U_{R}^{T} M_{\nu}^{(k)} U_{R}=\left(\begin{array}{ccc}
M_{1}^{(\kappa)} & 0 & 0 \\
0 & M_{2}^{(\kappa)} & 0 \\
0 & 0 & M_{3}^{(\kappa)}
\end{array}\right), \quad U_{R}=U_{\chi}=\left(\begin{array}{ccc}
-\frac{1}{\sqrt{3}} & -\frac{1}{\sqrt{2}} & -\frac{1}{\sqrt{6}} \\
-\frac{1}{\sqrt{3}} & \frac{1}{\sqrt{2}} & -\frac{1}{\sqrt{6}} \\
\frac{1}{\sqrt{3}} & 0 & -\sqrt{\frac{2}{3}}
\end{array}\right), \quad k=2,3
$$

where the sterile neutrino masses are given by:

$$
\begin{aligned}
& M_{1}^{(2)}=\left|\frac{\sqrt{3}\left(h_{\chi}^{(L)}\right)^{2} v_{\chi}^{2}}{2 h_{1 N} v_{\xi}}(X-2 Y)\right|, \quad M_{2}^{(2)}=M_{3}^{(2)}=\left|\frac{\sqrt{3}\left(h_{\chi}^{(L)}\right)^{2} v_{\chi}^{2}}{2 h_{1 N} v_{\xi}}(X+Y)\right|, \\
& M_{1}^{(3)}=\left|h_{1 N} \frac{v_{\xi}}{\sqrt{3}}(1-2 x)\right|, \quad M_{2}^{(3)}=M_{3}^{(3)}=\left|h_{1 N} \frac{v_{\xi}}{\sqrt{3}}(1+x)\right|,
\end{aligned}
$$

which implies that the heavy and very heavy sterile neutrino spectrum includes two degenerates heavy neutrino and one light neutrino states.

Furthermore, as follows from Eq. (34) and the relation $\left|\left(B_{2}\right)_{i j}\right| \sim 10^{-5}<<\left|\left(B_{1}\right)_{i j}\right| \sim 10^{-3}(i, j=1,2,3)$ given by Eq. (42), we can connect the neutrino fields $\nu_{L}=\left(\nu_{1 L}, \nu_{2 L}, \nu_{3 L}\right)^{T}, \nu_{R}^{C}=\left(\nu_{1 R}^{C}, \nu_{2 R}^{C}, \nu_{3 R}^{C}\right)$ and $N_{R}^{C}=\left(N_{1 R}^{C}, N_{2 R}^{C}, N_{3 R}^{C}\right)$ with the neutrino mass eigenstates by the following approximate relations:

$$
\left(\begin{array}{c}
\nu_{L} \\
\nu_{R}^{C} \\
N_{R}^{C}
\end{array}\right) \simeq\left(\begin{array}{c}
V_{\nu} \xi_{L}^{(1)} \\
U_{R} \xi_{L}^{(2)}+B_{1} U_{R} \xi_{L}^{(3)} \\
U_{R} \xi_{L}^{(3)}-B_{1}^{\dagger} U_{R} \xi_{L}^{(2)}
\end{array}\right), \quad \xi_{L}^{(j)}=\left(\begin{array}{c}
\xi_{1 L}^{(j)} \\
\xi_{2 L}^{(j)} \\
\xi_{3 L}^{(j)}
\end{array}\right), \quad j=1,2,3 .
$$


where $\xi_{k L}^{(1)}, \xi_{k L}^{(2)}$ and $\xi_{k L}^{(3)}(k=1,2,3)$ are the light active, heavy sterile and very heavy sterile neutrinos, respectively. As previously mentioned, the heavy sterile neutrinos have $\mathrm{MeV}$ scale masses and thus correspond to dark matter candidates. Furthermore, we assume that the lightest of the very heavy sterile neutrinos, i.e., $\xi_{1 L}^{(3)}$ has a TeV scale mass and thus corresponds to a candidate for detection at the LHC.

\section{QUARK MASSES AND MIXING.}

From Eq. (12) and taking into account that the VEV pattern of the $S U(3)_{L}$ singlet scalar fields is described by Eq. (14), with the nonvanishing VEVs set to be equal to $\lambda \Lambda$ (being $\Lambda$ the cutoff of our model) as shown in Eq. (15), it follows that the SM quark mass matrices have the form:

$$
M_{U}=\left(\begin{array}{ccc}
a_{11}^{(U)} \lambda^{4} & a_{12}^{(U)} \lambda^{3} & a_{13}^{(U)} \lambda^{2} \\
a_{21}^{(U)} \lambda^{3} & a_{22}^{(U)} \lambda^{2} & a_{23}^{(U)} \lambda \\
a_{31}^{(U)} \lambda^{2} & a_{32}^{(U)} \lambda & a_{33}^{(U)}
\end{array}\right) \frac{v}{\sqrt{2}}, \quad \quad M_{D}=\left(\begin{array}{ccc}
a_{11}^{(D)} \lambda^{7} & a_{12}^{(D)} \lambda^{6} & a_{13}^{(D)} \lambda^{5} \\
a_{21}^{(D)} \lambda^{6} & a_{22}^{(D)} \lambda^{5} & a_{23}^{(D)} \lambda^{4} \\
a_{31}^{(D)} \lambda^{5} & a_{32}^{(D)} \lambda^{4} & a_{33}^{(U)} \lambda^{3}
\end{array}\right) \frac{v}{\sqrt{2}},
$$

where $\lambda=0.225$ is one of the Wolfenstein parameters, $v=246 \mathrm{GeV}$ the scale of electroweak symmetry breaking and $a_{i j}^{(U, D)}(i, j=1,2,3)$ are $\mathcal{O}(1)$ parameters given by the following relations:

$$
\begin{aligned}
a_{n j}^{(U)}=y_{n j}^{(U)} \frac{v_{\rho}}{v} e^{i(6-j-n) \phi}, & a_{3 j}^{(U)}=y_{3 j}^{(U)} \frac{v_{\eta}}{v} e^{i(3-j) \phi}, & \\
a_{n j}^{(D)}=y_{n j}^{(D)} \frac{v_{\eta}}{v} e^{i(6-j-n) \phi}, & a_{3 j}^{(D)}=y_{3 j}^{(D)} \frac{v_{\rho}}{v} e^{i(3-j) \phi}, & n=1,2, \quad j=1,2,3 .
\end{aligned}
$$

Furthermore, the exotic quark masses read:

$$
m_{T}=y^{(T)} \frac{v_{\chi}}{\sqrt{2}}, \quad m_{J^{1}}=y_{1}^{(J)} \frac{v_{\chi}}{\sqrt{2}}=\frac{y_{1}^{(J)}}{y^{(T)}} m_{T}, \quad m_{J^{2}}=y_{2}^{(J)} \frac{v_{\chi}}{\sqrt{2}}=\frac{y_{2}^{(J)}}{y^{(T)}} m_{T} .
$$

Since the charged fermion mass and quark mixing pattern arises from the breaking of the $Z_{2} \otimes Z_{3} \otimes Z_{14}$ discrete group, we asssume an approximate universality in the dimensionless SM quark Yukawa couplings, as follows:

$$
\begin{aligned}
& a_{11}^{(U)}=a_{1}^{(U)} e^{i \phi_{1}}, \quad a_{22}^{(U)}=a_{2}^{(U)}, \quad a_{33}^{(U)}=a_{3}^{(U)}, \\
& a_{12}^{(U)}=a_{1}^{(U)}\left(1-\frac{\lambda^{2}}{2}\right)^{-\frac{3}{2}} e^{i \phi_{2}}, \quad a_{13}^{(U)}=a_{2}^{(U)}\left(1-\frac{\lambda^{2}}{2}\right)^{-\frac{3}{2}} e^{i \phi_{2}}, \quad a_{23}^{(U)}=\left|a_{13}^{(U)}\right|\left(1-\frac{\lambda^{2}}{2}\right)^{-\frac{3}{2}}, \\
& a_{11}^{(D)}=a_{22}^{(D)}\left(1-\frac{\lambda^{2}}{2}\right)^{-2}, \quad a_{23}^{(D)}=a_{33}^{(D)}\left(1-\frac{\lambda^{2}}{2}\right)^{-\frac{1}{2}}, \quad a_{i j}^{(U, D)}=a_{j i}^{(U, D)}, \quad i, j=1,2,3,
\end{aligned}
$$

It is noteworthy that exact universality in the dimensionless quark Yukawa couplings predicts massless up, down, strange and charm quarks, in contradiction with the experimental data on quark masses. Consequently, to generate these masses, the universality in the quark Yukawa couplings has to be broken. Besides that, for simplicity, we assume that the complex phase responsible for CP violation in the quark sector only arises from up type quark Yukawa terms, as indicated by Eq. (66). In addition, for the sake of simplicity, we fix $a_{3}^{(U)}=1$, which is suggested by naturalness arguments. Therefore, the SM quark mass matrices are given by:

$$
\begin{gathered}
M_{U}=\left(\begin{array}{ccc}
a_{1}^{(U)} \lambda^{4} e^{i \phi_{1}} & a_{1}^{(U)}\left(1-\frac{\lambda^{2}}{2}\right)^{-\frac{3}{2}} \lambda^{3} e^{i \phi_{2}} & a_{2}^{(U)}\left(1-\frac{\lambda^{2}}{2}\right)^{-\frac{3}{2}} \lambda^{2} e^{i \phi_{2}} \\
a_{1}^{(U)}\left(1-\frac{\lambda^{2}}{2}\right)^{-\frac{3}{2}} \lambda^{3} e^{i \phi_{2}} & a_{2}^{(U)} \lambda^{2} & a_{2}^{(U)}\left(1-\frac{\lambda^{2}}{2}\right)^{-3} \lambda \\
a_{2}^{(U)}\left(1-\frac{\lambda^{2}}{2}\right)^{-\frac{3}{2}} \lambda^{2} e^{i \phi_{2}} & a_{2}^{(U)}\left(1-\frac{\lambda^{2}}{2}\right)^{-3} \lambda & a_{3}^{(U)}
\end{array}\right) \frac{v}{\sqrt{2}}, \\
M_{D}=\left(\begin{array}{ccc}
a_{22}^{(D)}\left(1-\frac{\lambda^{2}}{2}\right)^{-2} \lambda^{7} & a_{12}^{(D)} \lambda^{6} & a_{13}^{(D)} \lambda^{5} \\
a_{12}^{(D)} \lambda^{6} & a_{22}^{(D)} \lambda^{5} & a_{33}^{(D)}\left(1-\frac{\lambda^{2}}{2}\right)^{-\frac{1}{2}} \lambda^{4} \\
a_{13}^{(D)} \lambda^{5} & a_{33}^{(D)}\left(1-\frac{\lambda^{2}}{2}\right)^{-\frac{1}{2}} \lambda^{4} & a_{33}^{(D)} \lambda^{3}
\end{array}\right) \frac{v}{\sqrt{2}}
\end{gathered}
$$


Let's recall that the quark sector has 10 effective parameters, i.e, $\lambda, a_{3}^{(U)}, a_{1}^{(U)}, a_{2}^{(U)}, a_{22}^{(D)}, a_{12}^{(D)}, a_{13}^{(D)}, a_{33}^{(D)}$ and the phases $\phi_{1}$ and $\phi_{2}$ to describe the quark mass and mixing pattern, which is determined by 10 observables. Nevertheless, not all these effective parameters are free since the parameters $\lambda$ and $a_{3}^{(U)}$ are fixed while the remaining 8 parameters are adjusted to reproduce the physical observables in the quark sector, i.e., 6 quark masses and 4 quark mixing parameters. The results shown in Table III correspond to the following best-fit values:

$$
\begin{aligned}
& a_{1}^{(U)} \simeq 0.64, \quad a_{2}^{(U)} \simeq 0.77, \quad \phi_{1} \simeq-9.03^{\circ}, \quad \phi_{2} \simeq-4.53^{\circ}, \\
& a_{22}^{(D)} \simeq 2.03, \quad a_{12}^{(D)} \simeq 1.75, \quad a_{13}^{(D)} \simeq 1.15, \quad a_{33}^{(D)} \simeq 1.40 .
\end{aligned}
$$

\begin{tabular}{c|l|l}
\hline \hline Observable & Model value & Experimental value \\
\hline$m_{u}(\mathrm{MeV})$ & 1.59 & $1.45_{-0.45}^{+0.56}$ \\
\hline$m_{c}(\mathrm{MeV})$ & 673 & $635 \pm 86$ \\
\hline$m_{t}(\mathrm{GeV})$ & 180 & $172.1 \pm 0.6 \pm 0.9$ \\
\hline$m_{d}(\mathrm{MeV})$ & 2.9 & $2.9_{-0.4}^{+0.5}$ \\
\hline$m_{s}(\mathrm{MeV})$ & 59.7 & $57.7_{-15.7}^{+16.8}$ \\
\hline$m_{b}(\mathrm{GeV})$ & 2.98 & $2.82_{-0.04}^{+0.09}$ \\
\hline$\left|V_{u d}\right|$ & 0.975 & $0.97427 \pm 0.00015$ \\
\hline$\left|V_{u s}\right|$ & 0.224 & $0.22534 \pm 0.00065$ \\
\hline$\left|V_{u b}\right|$ & 0.0036 & $0.00351_{-0.00014}^{+0.00015}$ \\
\hline$\left|V_{c d}\right|$ & 0.224 & $0.22520 \pm 0.00065$ \\
\hline$\left|V_{c s}\right|$ & 0.9736 & $0.97344 \pm 0.00016$ \\
\hline$\left|V_{c b}\right|$ & 0.0433 & $0.0412_{-0.0005}^{+0.0011}$ \\
\hline$\left|V_{t d}\right|$ & 0.00853 & $0.00867_{-0.00031}^{+0.00029}$ \\
\hline$\left|V_{t s}\right|$ & 0.0426 & $0.0404_{-0.0005}^{+0.0011}$ \\
\hline$\left|V_{t b}\right|$ & 0.999057 & $0.999146_{-0.000046}^{+0.000021}$ \\
\hline$J$ & $2.98 \times 10^{-5}$ & $\left(2.96_{-0.16}^{+0.20}\right) \times 10^{-5}$ \\
\hline$\delta$ & $61^{\circ}$ & $68^{\circ}$ \\
\hline \hline
\end{tabular}

Table III: Model and experimental values of the quark masses and CKM parameters.

The obtained and experimental values of the quark masses, CKM matrix elements, Jarlskog invariant $J$ and CP violating phase $\delta$ are reported in Table III. We use the experimental values of the quark masses at the $M_{Z}$ scale, from Ref. [52] (which are similar to those in [53]), whereas we use the experimental values of the CKM parameters from Ref. [6]. The obtained values of the quark masses and CKM parameters are in excellent agreement with the experimental data, as indicated by Table III

\section{CONCLUSIONS}

In this paper we present an extension of the minimal 331 model with $\beta=-\frac{1}{\sqrt{3}}$, based on the extended $S U(3)_{C} \otimes$ $S U(3)_{L} \otimes U(1)_{X} \otimes T_{7} \otimes Z_{2} \otimes Z_{3} \otimes Z_{14}$ symmetry. Our economical $T_{7}$ flavor 331 model, which at low energies reduces to the minimal 331 model with $\beta=-\frac{1}{\sqrt{3}}$, is compatible with the experimental data on fermion masses and mixing. The model has in total 16 effective free parameters, which are fitted to reproduce the experimental values of the 18 physical observables in the quark and lepton sectors. The $T_{7}$ and $Z_{3}$ symmetries reduce the number of model parameters. In particular, the $Z_{3}$ symmetry determines the allowed entries of the neutrino mass matrix and decouples the SM quarks from the exotic quarks. The $Z_{2}$ symmetry generates the hierarchy between SM up and SM down type quark masses. We assumed that the $S U(3)_{L}$ scalar singlets having Yukawa interactions with the right handed Majorana neutrinos acquire VEVs at very high scale, then providing very large masses to these Majorana neutrinos, and thus giving rise to a double seesaw mechanism of active neutrino masses. Consequently, the neutrino spectrum includes very light active neutrinos as well as heavy and very heavy sterile neutrinos. We find that the heavy and very heavy sterile neutrinos have masses at the $\sim \mathrm{MeV}$ and $\sim \mathrm{TeV}$ scales, respectively. Thus, the MeV scale sterile neutrinos of our model correspond to dark matter candidates. The smallness of the active neutrino masses is attributed to their 
scaling with inverse powers of the high energy cutoff $\Lambda$ as well as well as by their quadratic dependence on the very small VEV of the $Z_{2} \otimes Z_{3} \otimes Z_{14}$ neutral, $S U(3)_{L}$ singlet and $T_{7}$ antitriplet scalar field $\zeta$. The observed hierarchy of charged fermion masses and quark mixing matrix elements arises from the breaking of the $Z_{2} \otimes Z_{3} \otimes Z_{14}$ discrete group at a very high energy. The tau, muon and electron masses arise from effective seven, nine and twelve dimensional Yukawa operators, respectively. We find for the scale of these operators the estimate $\Lambda \sim 10^{5} \mathrm{TeV}$. The complex phase responsible for $\mathrm{CP}$ violation in the quark sector has been assumed to come from up-type quark Yukawa terms. The model predicts an effective Majorana neutrino mass, relevant for neutrinoless double beta decay, with values $m_{\beta \beta}=$ 3 and $40 \mathrm{meV}$, for the normal and the inverted neutrino spectrum, respectively. In the latter case our prediction is within the declared reach of the next generation bolometric CUORE experiment [47] or, more realistically, of the next-to-next generation tone-scale $0 \nu \beta \beta$-decay experiments. Furthermore, a vanishing leptonic Dirac CP violating phase is predicted in our model.

\section{Acknowledgments}

We are very grateful to Fredy Ochoa for careful reading of the manuscript and for valuable discussions. A.E.C.H was supported by Fondecyt (Chile), Grant No. 11130115 and by DGIP internal Grant No. 111458. R.M. was supported by El Patrimonio Autónomo Fondo Nacional de Financiamiento para la Ciencia, la Tecnología y la Innovación Fransisco José de Caldas programme of COLCIENCIAS in Colombia.

\section{Appendix A: The product rules for $T_{7}$}

The group $T_{7}$, which is a subgroup of $S U(3)$ and $\Delta\left(3 N^{2}\right)$ with $N=7$, has 21 elements, is isomorphic to $Z_{7} \rtimes Z_{3}$ and contains five irreducible representations, i.e., one triplet $\mathbf{3}$, one antitriplet $\overline{\mathbf{3}}$ and three singlets $\mathbf{1}_{0}, \mathbf{1}_{1}$ and $\mathbf{1}_{2}$ [10]. The discrete group $T_{7}$ is the minimal non-Abelian discrete group having a complex triplet. The triplet and antitriplet irreducible representations are defined as [10]:

$$
\mathbf{3} \equiv\left(\begin{array}{l}
x_{1} \\
x_{2} \\
x_{4}
\end{array}\right), \quad \overline{\mathbf{3}} \equiv\left(\begin{array}{l}
x_{-1} \\
x_{-2} \\
x_{-4}
\end{array}\right)=\left(\begin{array}{l}
x_{6} \\
x_{5} \\
x_{3}
\end{array}\right) .
$$

The product rules for triplet and antitriplet tensor irreducible representations are given by:

$$
\begin{aligned}
\left(\begin{array}{l}
x_{1} \\
x_{2} \\
x_{4}
\end{array}\right)_{\mathbf{3}} \otimes\left(\begin{array}{l}
y_{1} \\
y_{2} \\
y_{4}
\end{array}\right)_{\mathbf{3}}=\left(\begin{array}{l}
x_{2} y_{4} \\
x_{4} y_{1} \\
x_{1} y_{2}
\end{array}\right)_{\overline{\mathbf{3}}} \oplus\left(\begin{array}{l}
x_{4} y_{2} \\
x_{1} y_{4} \\
x_{2} y_{1}
\end{array}\right)_{\overline{\mathbf{3}}} \oplus\left(\begin{array}{l}
x_{4} y_{4} \\
x_{1} y_{1} \\
x_{2} y_{2}
\end{array}\right)_{\mathbf{3}}, \\
\left(\begin{array}{l}
x_{6} \\
x_{5} \\
x_{3}
\end{array}\right)_{\overline{\mathbf{3}}} \otimes\left(\begin{array}{l}
y_{6} \\
y_{5} \\
y_{3}
\end{array}\right)_{\overline{\mathbf{3}}}= \\
\left(\begin{array}{l}
x_{1} \\
x_{2} \\
x_{4}
\end{array}\right)_{\mathbf{3}} \otimes\left(\begin{array}{l}
x_{5} y_{3} \\
x_{3} y_{6} \\
x_{6} y_{5}
\end{array}\right)_{\mathbf{3}} \oplus\left(\begin{array}{l}
x_{3} y_{5} \\
x_{6} y_{3} \\
x_{5} y_{6}
\end{array}\right)_{\mathbf{3}} \oplus\left(\begin{array}{l}
x_{3} y_{3} \\
x_{6} y_{6} \\
x_{5} y_{5}
\end{array}\right)_{\overline{\mathbf{3}}}, \\
=\left(\begin{array}{l}
x_{2} y_{6} \\
x_{4} y_{5} \\
x_{1} y_{3}
\end{array}\right)_{\mathbf{3}} \oplus\left(\begin{array}{l}
x_{1} y_{5} \\
x_{2} y_{3} \\
x_{4} y_{6}
\end{array}\right)_{\overline{\mathbf{3}}} \\
\oplus \sum_{k=0,1,2}\left(x_{1} y_{6}+\omega^{k} x_{2} y_{5}+\omega^{2 k} x_{4} y_{3}\right)_{\mathbf{1}_{k}} .
\end{aligned}
$$

Whereas the tensor products between singlets are:

$$
\begin{aligned}
& \left.\left.(x)_{\mathbf{1}_{0}}(y)_{\mathbf{1}_{0}}=(x)_{\mathbf{1}_{1}}(y)\right)_{\mathbf{1}_{2}}=(x)\right)_{\mathbf{1}_{2}}(y)_{\mathbf{1}_{1}}=(x y)_{\mathbf{1}_{0}}, \\
& (x)_{\mathbf{1}_{1}}(y)_{\mathbf{1}_{1}}=(x y)_{\mathbf{1}_{2}} \\
& (x)_{\mathbf{1}_{2}}(y)_{\mathbf{1}_{2}}=(x y)_{\mathbf{1}_{1}} .
\end{aligned}
$$


The product rules between triplets and singlets satisfy the relations:

$$
(y)_{\mathbf{1}_{k}} \otimes\left(\begin{array}{l}
x_{1(6)} \\
x_{2(5)} \\
x_{4(3)}
\end{array}\right)_{\mathbf{3}(\overline{\mathbf{3}})}=\left(\begin{array}{l}
y x_{1(6)} \\
y x_{2(5)} \\
y x_{4(3)}
\end{array}\right)_{\mathbf{3}(\overline{\mathbf{3}})} .
$$

where $\omega=e^{i \frac{2 \pi}{3}}$. The representation $\mathbf{1}_{0}$ is trivial, while the non-trivial $\mathbf{1}_{1}$ and $\mathbf{1}_{2}$ are complex conjugate to each other. Some reviews of discrete symmetries in particle physics are found in Refs. [8 10, 54].

[1] G. Aad et al. [The ATLAS Collaboration], "Observation of a new particle in the search for the Standard Model Higgs boson with the ATLAS detector at the LHC,", Phys. Lett. B 716 (2012) 1 arXiv:hep-ex/1207.7214.

[2] S. Chatrchyan et al. [The CMS Collaboration], "Observation of a new boson at a mass of 125 GeV with the CMS experiment at the LHC," , Phys. Lett. B 716, 30 (2012) arXiv:hep-ex/1207.7235.

[3] T. Aaltonen et al. [CDF and D0 Collaborations], "Evidence for a particle produced in association with weak bosons and decaying to a bottom-antibottom quark pair in Higgs boson searches at the Tevatron,", Phys. Rev. Lett. 109 (2012) 071804, [ arXiv:hep-ex/1207.6436].

[4] The CMS Collaboration, "Observation of a new boson with a mass near 125 GeV," CMS-PAS-HIG-12-020.

[5] S.L. Glashow, Nucl. Phys. 22, 579 (1961); S. Weinberg, Phys. Rev. Lett. 19, 1264 (1967); A. Salam, in Elementary Particle Theory: Relativistic Groups and Analyticity (Nobel Symposium No. 8), edited by N.Svartholm (Almqvist and Wiksell, Stockholm, 1968), p. 367.

[6] K. A. Olive et al. [Particle Data Group Collaboration], Chin. Phys. C 38, 090001 (2014).

[7] J. K. Mizukoshi, C. A. de S.Pires, F. S. Queiroz and P. S. Rodrigues da Silva, Phys. Rev. D 83, 065024 (2011) arXiv:1010.4097 [hep-ph]]; C. Kelso, C. A. de S. Pires, S. Profumo, F. S. Queiroz and P. S. Rodrigues da Silva, Eur. Phys. J. C 74, 2797 (2014) arXiv:1308.6630 [hep-ph]]; S. Profumo and F. S. Queiroz, Eur. Phys. J. C 74, 2960 (2014) arXiv:1307.7802 [hep-ph]]; C. Kelso, H. N. Long, R. Martinez and F. S. Queiroz, Phys. Rev. D 90, 113011 (2014) arXiv:1408.6203 [hep-ph]]; R. Martinez, J. Nisperuza, F. Ochoa and J. P. Rubio, Phys. Rev. D 90, 095004 (2014) arXiv:1408.5153 [hep-ph]]; H. Okada and Y. Orikasa, Phys. Rev. D 90, no. 7, 075023 (2014) arXiv:1407.2543 [hepph]]; H. Hatanaka, K. Nishiwaki, H. Okada and Y. Orikasa, arXiv:1412.8664 [hep-ph]; P. V. Dong, C. S. Kim, D. V. Soa and N. T. Thuy, arXiv:1501.04385 [hep-ph]; I. d. M. Varzielas, O. Fischer and V. Maurer, arXiv:1504.03955 [hep-ph]; A. Ahmed, B. Grzadkowski, J. F. Gunion and Y. Jiang, arXiv:1504.03706 [hep-ph].

[8] S. F. King and C. Luhn, arXiv:1301.1340 [hep-ph].

[9] G. Altarelli and F. Feruglio, Rev. Mod. Phys. 82 (2010) 2701 arXiv:1002.0211 [hep-ph]].

[10] H. Ishimori, T. Kobayashi, H. Ohki, Y. Shimizu, H. Okada and M. Tanimoto, Prog. Theor. Phys. Suppl. 183 (2010) 1 arXiv:1003.3552 [hep-th]].

[11] T. Kobayashi, H. P. Nilles, F. Ploger, S. Raby and M. Ratz, Nucl. Phys. B 768, 135 (2007) hep-ph/0611020; T. Kobayashi, Y. Omura and K. Yoshioka, Phys. Rev. D 78, 115006 (2008) arXiv:0809.3064 [hep-ph]]; H. Abe, K. S. Choi, T. Kobayashi and H. Ohki, Nucl. Phys. B 820, 317 (2009) arXiv:0904.2631 [hep-ph]]; M. Berasaluce-Gonzalez, L. E. Ibanez, P. Soler and A. M. Uranga, JHEP 1112, 113 (2011) arXiv:1106.4169 [hep-th]]; F. Beye, T. Kobayashi and S. Kuwakino, Phys. Lett. B 736, 433 (2014) arXiv:1406.4660 [hep-th]].

[12] F. Pisano and V. Pleitez, Phys. Rev. D46, 410 (1992); Nguyen Tuan Anh, Nguyen Anh Ky, Hoang Ngoc Long, Int. J. Mod. Phys. A16, 541 (2001).

[13] P.H. Frampton, Phys. Rev. Lett. 69, 2889 (1992).

[14] R. Foot, H.N. Long and T.A. Tran, Phys. Rev. D50, R34 (1994); H.N. Long, ibid. 53, 437 (1996); 54, 4691 (1996); Mod. Phys. Lett. A 13, 1865 (1998).

[15] R. A. Diaz, R. Martinez, J. Mira and J. A. Rodriguez, Phys. Lett. B 552, 287 (2003) hep-ph/0208176]; Rodolfo A. Diaz, R. Martinez, F. Ochoa, Phys. Rev. D69, 095009 (2004); D72, 035018 (2005); Fredy Ochoa, R. Martinez, Phys. Rev D72, 035010 (2005); A. Carcamo, R. Martinez and F. Ochoa, Phys. Rev. D73, 035007 (2006); C. Alvarado, R. Martínez and F. Ochoa, Phys. Rev. D86, 025027 (2012) A. E. Cárcamo Hernández, R. Martínez and F. Ochoa, Phys. Rev. D 87 (2013) 075009 arXiv:1302.1757 [hep-ph]]; H. Okada, N. Okada and Y. Orikasa, arXiv:1504.01204 [hep-ph]; A. E. Cárcamo Hernández and I. Nisandzic, arXiv:1512.07165 [hep-ph]; S. M. Boucenna, S. Morisi and A. Vicente, arXiv:1512.06878 [hep-ph].

[16] J.S. Bell, R. Jackiw, Nuovo Cim. A60, 47 (1969); S.L. Adler, Phys. Rev. 177, 2426 (1969); D.J. Gross, R. Jackiw, Phys.Rev. D6, 477 (1972). H. Georgi and S. L. Glashow, Phys. Rev. D6, 429 (1972); S. Okubo, Phys. Rev. D16, 3528 (1977); J. Banks and H. Georgi, Phys. Rev. 14, 1159 (1976).

[17] F. P. An et al. (DAYA-BAY Collaboration), Phys. Rev. Lett. 108, 171803 (2012).

[18] K. Abe et al. (T2K Collaboration), Phys. Rev. Lett. 107, 041801 (2011).

[19] P. Adamson et al. (MINOS Collaboration), Phys. Rev. Lett. 107, 181802 (2011).

[20] Y. Abe et al. (DOUBLE-CHOOZ Collaboration), Phys. Rev. Lett. 108, 131801 (2012).

[21] J. K. Ahn et al. (RENO Collaboration), Phys. Rev. Lett. 108, 191802 (2012). 
[22] C. Kraus, B. Bornschein, L. Bornschein, J. Bonn, B. Flatt, A. Kovalik, B. Ostrick and E. W. Otten et al., Eur. Phys. J. C 40, 447 (2005) hep-ex/0412056.

[23] EXO Collaboration, M. Auger et al., Phys.Rev.Lett. 109, 032505 (2012), arXiv:1205.5608; GERDA Collaboration, I. Abt et al., (2004), arXiv:hep-ex/0404039. EXO-200 Collaboration, D. Auty, Recontres de Moriond, http://moriond.in2p3.fr/ (2013); O. Cremonesi and M. Pavan, arXiv:1310.4692 [physics.ins-det]; W. Rodejohann, J. Phys. G 39, 124008 (2012) arXiv:1206.2560 [hep-ph]]; A. Barabash, (2012), arXiv:1209.4241 F. F. Deppisch, M. Hirsch and H. Pas, J. Phys. G 39, 124007 (2012) arXiv:1208.0727 [hep-ph]]; A. Giuliani and A. Poves, Adv. High Energy Phys. 2012, 857016 (2012); S. M. Bilenky and C. Giunti, arXiv:1411.4791 [hep-ph].

[24] P. A. R. Ade et al. [Planck Collaboration], Astron. Astrophys. 571, A16 (2014) arXiv:1303.5076 [astro-ph.CO]].

[25] D. V. Forero, M. Tortola and J. W. F. Valle, Phys. Rev. D 90, no. 9, 093006 (2014) arXiv:1405.7540 [hep-ph]].

[26] E. Ma and G. Rajasekaran, Phys. Rev. D 64, 113012 (2001); G. Altarelli and F. Feruglio, Nucl. Phys. B 741, 215 (2006); S. L. Chen, M. Frigerio and E. Ma, Nucl. Phys. B 724, 423 (2005); A. Zee, Phys. Lett. B 630, 58 (2005); M. Hirsch, S. Morisi and J. W. F. Valle, Phys. Lett. B 679, 454 (2009) arXiv:0905.3056 [hep-ph]]; T Fukuyama, H. Sugiyama and K. Tsumura, Phys. Rev. D 83, 056016 (2011) arXiv:1012.4886 [hep-ph]]; P. V. Dong, L. T. Hue, H. N. Long and D. V. Soa, Phys. Rev. D 81, 053004 (2010) arXiv:1001.4625 [hep-ph]]; Miguel D. Campos, A. E. Cárcamo Hernández, S. G. Kovalenko, I. Schmidt and Erik Schumacher, Phys. Rev. D 90016006 (2014) arXiv:1403.2525 [hep-ph]]; A. C. B. Machado, J. C. Montero and V. Pleitez, Phys. Lett. B 697, 318 (2011) arXiv:1011.5855 [hep-ph]]; P. S. Bhupal Dev, R. N. Mohapatra and M. Severson, Phys. Rev. D 84, 053005 (2011) arXiv:1107.2378 [hep-ph]]; P. S. Bhupal Dev, B. Dutta, R. N. Mohapatra and M. Severson, Phys. Rev. D 86, 035002 (2012) arXiv:1202.4012 [hep-ph]]; Y. Kajiyama, H. Okada and K. Yagyu, JHEP 1310, 196 (2013) arXiv:1307.0480 [hep-ph]]; I. de Medeiros Varzielas and Luís Lavoura, J. Phys. G 40, 085002 (2013) arXiv:1212.3247 [hep-ph]]; P. M. Ferreira, L. Lavoura and P. O. Ludl, arXiv:1306.1500 [hep-ph]; M. C. Chen, J. Huang, J. M. O'Bryan, A. M. Wijangco and F. Yu, JHEP 1302, 021 (2013) doi:10.1007/JHEP02(2013)021 arXiv:1210.6982 [hepph]]; A. E. Cárcamo Hernández, I. d. M. Varzielas, S. G. Kovalenko, H. Pas and I. Schmidt, Phys. Rev. D 88076014 (2013) arXiv:1307.6499 [hep-ph]]; Miguel D. Campos, A. E. Cárcamo Hérnandez, H. Pas and Erik Schumacher, arXiv:1408.1652 [hep-ph]; A. E. Cárcamo Hernández, S. G. Kovalenko and I. Schmidt, arXiv:1411.2913 [hep-ph]; A. E. Cárcamo Hernández, R. Martinez and F. Ochoa, arXiv:1309.6567 [hep-ph]; A. E. Cárcamo Hernández and R. Martinez, arXiv:1501.05937 [hepph]; M. Abbas and S. Khalil, Phys. Rev. D 91, no. 5, 053003 (2015) arXiv:1406.6716 [hep-ph]];

[27] F. Feruglio, C. Hagedorn, Y. Lin and L. Merlo, Nucl. Phys. B 775, 120 (2007); M. C. Chen and K. T. Mahanthappa, Phys. Lett. B 652, 34 (2007); S. Morisi, M. Nebot, K. M. Patel, E. Peinado and J. W. F. Valle, arXiv:1303.4394 [hep-ph]; Z. z. Xing, D. Yang, S. Zhou, [arXiv:hep-ph/1004.4234v2]; J. E. Kim, M. S. Seo, JHEP 1102 (2011) 097, [arXiv:hepph/1005.4684]; I. de Medeiros Varzielas and D. Pidt, arXiv:1307.0711 [hep-ph].

[28] S. Pakvasa and H. Sugawara, Phys. Lett. B 73 (1978) 61; E. Ma, Phys. Rev. D 61, 033012 (2000) arXiv:hep-ph/9909249; W. Grimus and L. Lavoura, JHEP 0508, 013 (2005) arXiv:hep-ph/0504153; P. V. Dong, H. N. Long, C. H. Nam and V. V. Vien, Phys. Rev. D 85, 053001 (2012) arXiv:1111.6360 [hep-ph]]; F. González Canales, A. Mondragón, M. Mondragón, U. J. Saldana Salazar and L. Velasco-Sevilla, Phys. Rev. D 88, 096004 (2013) arXiv:1304.6644 [hep-ph]]; Y. Kajiyama, H. Okada and K. Yagyu, arXiv:1309.6234 [hep-ph].

[29] A. E. Cárcamo Hernández, M.E. Cataño and R. Martinez, Phys. Rev. D 90, 073001 (2014) arXiv:1407.5217 [hep-ph]].

[30] A. E. Cárcamo Hernández, R. Martinez and Jorge Nisperuza, Eur. Phys. J. C 75, no. 2, 72 (2015) arXiv:1401.0937 [hep-ph]].

[31] A. E. Cárcamo Hernández, I. de Medeiros Varzielas and E. Schumacher, arXiv:1509.02083 [hep-ph].

[32] A. E. Cárcamo Hernández, I. de Medeiros Varzielas and Nicolás A. Neill, arXiv:1511.07420 [hep-ph].

[33] I. d. M. Varzielas and D. Pidt, arXiv:1307.0711 [hep-ph]; G. Bhattacharyya, I.M. Varzielas and P. Leser, Phys. Rev. Lett. 109, 241603 (2012) arXiv:1210.0545 [hep-ph]]; C. C. Nishi, arXiv:1306.0877 [hep-ph]; I. de Medeiros Varzielas, JHEP 1508, 157 (2015) doi:10.1007/JHEP08(2015)157 arXiv:1507.00338 [hep-ph]]; G. C. Branco, I. de Medeiros Varzielas and S. F. King, Nucl. Phys. B 899, 14 (2015) doi:10.1016/j.nuclphysb.2015.07.024 arXiv:1505.06165 [hep-ph]]; P. Chen, G. J. Ding, A. D. Rojas, C. A. Vaquera-Araujo and J. W. F. Valle, arXiv:1509.06683 [hep-ph]; V. V. Vien, A. E. Cárcamo Hernández and H. N. Long, arXiv:1601.03300 [hep-ph]; A. E. Cárcamo Hernández, H. N. Long and V. V. Vien, arXiv:1601.05062 [hep-ph].

[34] P. H. Frampton, T. W. Kephart and S. Matsuzaki, Phys. Rev. D 78, 073004 (2008) arXiv:0807.4713 [hep-ph]]; M. C. Chen, K. T. Mahanthappa and F. Yu, Phys. Rev. D 81, 036004 (2010) doi:10.1103/PhysRevD.81.036004 arXiv:0907.3963 [hepph]]; P. H. Frampton, C. M. Ho and T. W. Kephart, Phys. Rev. D 89, 027701 (2014) arXiv:1305.4402 [hep-ph]]; M. C. Chen, K. T. Mahanthappa, A. Meroni and S. T. Petcov, arXiv:1109.0731 [hep-ph]; I. Girardi, A. Meroni, S. T. Petcov and M. Spinrath, JHEP 1402, 050 (2014) arXiv:1312.1966 [hep-ph]]; M. C. Chen, J. Huang, K. T. Mahanthappa and A. M. Wijangco, JHEP 1310, 112 (2013) doi:10.1007/JHEP10(2013)112 arXiv:1307.7711 [hep-ph]].

[35] C. Luhn, S. Nasri and P. Ramond, Phys. Lett. B 652, 27 (2007) arXiv:0706.2341 [hep-ph]]; C. Hagedorn, M. A. Schmidt and A. Y. Smirnov, Phys. Rev. D 79, 036002 (2009) arXiv:0811.2955 [hep-ph]]; C. Luhn, K. M. Parattu and A. Wingerter, JHEP 1212, 096 (2012) arXiv:1210.1197 [hep-ph]]; H. Ishimori, S. Khalil and E. Ma, Phys. Rev. D 86, 013008 (2012) arXiv:1204.2705 [hep-ph]]; V. V. Vien and H. N. Long, JHEP 1404, 133 (2014) arXiv:1402.1256 [hep-ph]]; C. Bonilla, S. Morisi, E. Peinado and J. W. F. Valle, arXiv:1411.4883 [hep-ph]; Carolina Arbelaéz, A. E. Cárcamo Hernández, Sergey Kovalenko and Iván Schmidt, arXiv:1507.03852 [hep-ph], to be published in Physical Review D.

[36] H. Fritzsch, Phys. Lett. B70, 436 (1977); H. Fritzsch, Phys. Lett. B73, 317 (1978); T.P. Cheng and M. Sher, Phys. Rev. D35, 3484 (1987); H. Fritzsch and J. Planck, Phys. Lett. B 237, 451 (1990); D. s. Du and Z. z. Xing, Phys. Rev. D 48, 2349 (1993); K. Matsuda and H. Nishiura, Phys. Rev. D74, 033014 (2006); A. E. Cárcamo Hernández, R. Martinez and J. A. Rodriguez, Eur. Phys. J. C50, 935 (2007); A. E. Cárcamo Hernández, R. Martinez and J. A. Rodriguez, AIP 
Conf. Proc. 1026 (2008) 272; H. Okada and K. Yagyu, Phys. Rev. D 89053008 (2014) arXiv:1311.4360 [hep-ph]]; A. E. Cárcamo Hernández and I. d. M. Varzielas, J. Phys. G 42, no. 6, 065002 (2015) doi:10.1088/0954-3899/42/6/065002 arXiv:1410.2481 [hep-ph]].

[37] R. Barbieri, G. R. Dvali, A. Strumia, Z. Berezhiani and L. J. Hall, Nucl. Phys. B 432, 49 (1994) arXiv:hep-ph/9405428; Z. Berezhiani, Phys. Lett. B 355, 481 (1995) arXiv:hep-ph/9503366; A. E. Cárcamo Hernández and Rakibur Rahman [arXiv:hep-ph/1007.0447], to be published in Revista Mexicana de Física.

[38] B. A. Dobrescu, Phys. Lett. B 461, 99 (1999) arXiv:hep-ph/9812349; G. Altarelli and F. Feruglio, Nucl. Phys. B 720 64 (2005) hep-ph/0504165; A. E. Cárcamo Hernández, Claudio. O. Dib, Nicolás Neill H and Alfonso R. Zerwekh, JHEP 1202 (2012) 132 [arXiv:hep-ph/1201.0878].

[39] K. S. Babu and R. N. Mohapatra, Phys. Rev. Lett. 74, 2418 (1995) arXiv:hep-ph/9410326.

[40] L. E. Ibanez and G. G. Ross, Phys. Lett. B 332, 100 (1994); P. Binetruy and P. Ramond, Phys. Lett. B 350, 49 (1995); Y. Nir, Phys. Lett. B 354, 107 (1995); V. Jain and R. Shrock, Phys. Lett. B 352, 83 (1995); E. Dudas, S. Pokorski and C. A. Savoy, Phys. Lett. B 356, 45 (1995); B 369, 255 (1996).

[41] M.E. Catano, R. Martinez and F. Ochoa, Phys. Rev. D 86, 073015 (2012) arXiv:1206.1966 [hep-ph]].

[42] A. C. B. Machado, J. C. Montero and V. Pleitez, Phys. Lett. B 697, 318 (2011) arXiv:1011.5855 [hep-ph]].

[43] M. Maniatis, A. von Manteuffel, O. Nachtmann and F. Nagel, Eur. Phys. J. C 48, 805 (2006) hep-ph/0605184.

[44] M. Auger et al. [EXO-200 Collaboration], Phys. Rev. Lett. 109, 032505 (2012) arXiv:1205.5608 [hep-ex]].

[45] I. Abt et al., hep-ex/0404039.

[46] K. H. Ackermann et al. [GERDA Collaboration], Eur. Phys. J. C 73, no. 3, 2330 (2013) arXiv:1212.4067 [physics.ins-det]].

[47] F. Alessandria et al., arXiv:1109.0494 [nucl-ex].

[48] A. Gando et al. [KamLAND-Zen Collaboration], Phys. Rev. C 85, 045504 (2012) arXiv:1201.4664 [hep-ex]].

[49] J. B. Albert et al. [EXO-200 Collaboration], Phys. Rev. D 90, no. 9, 092004 (2014) arXiv:1409.6829 [hep-ex]].

[50] C. E. Aalseth et al. [Majorana Collaboration], Nucl. Phys. Proc. Suppl. 217, 44 (2011) arXiv:1101.0119 [nucl-ex]].

[51] S. M. Bilenky and C. Giunti, Int. J. Mod. Phys. A 30, no. 04n05, 1530001 (2015) arXiv:1411.4791 [hep-ph]].

[52] K. Bora, J. Phys. 2, 2013 arXiv:1206.5909 [hep-ph]].

[53] Z. z. Xing, H. Zhang and S. Zhou, Phys. Rev. D 77, 113016 (2008) arXiv:0712.1419 [hep-ph]].

[54] P. Ramond, Group Theory: A Physicist's Survey, Cambridge University Press, UK (2010); C. Luhn, S. Nasri and P. Ramond, J. Math. Phys. 48, 123519 (2007) arXiv:0709.1447 [hep-th]]. 